\title{
Application of Shannon Entropy in the Construction of a Paraconsistent Model of the Atom
}

\author{
João Inácio da Silva Filho * \\ Laboratory of Applied Paraconsistent Logic, Santa Cecilia University \\ Oswaldo Cruz Street, 288, Santos City, SP, Brazil, 11045-000 \\ * Correspondence: inacio@unisanta.br
}

\begin{abstract}
In this work, we present a model of the atom that is based on a nonclassical logic called paraconsistent logic (PL), which has the main property of accepting the contradiction in logical interpretations without the conclusions being annulled. The proposed model is constructed with an extension of PL called paraconsistent annotated logic with annotation of two values (PAL2v), which is associated with an interlaced bilattice of four vertices. We use the logarithmic function of the Shannon entropy $H_{(\mathrm{s})}$ to construct the paraconsistent equations and thus adapt a probabilistic model for representations in quantum physics. Through analyses of the interlaced bilattice, comparative values are obtained for some of the phenomena and effects of quantum mechanics, such as superposition of states, quantum entanglement, wave functions, and equations that determine the energy levels of the layers of an atom. At the end of this article, we use the hydrogen atom as a basis of the representation of the PAL2v model, where the values of the energy levels in six orbital layers are obtained. As an example, we present a possible method of applying the PAL2 $\mathrm{v}$ model to the use of Raman spectroscopy signals in the detection of lubricating mineral oil quality.
\end{abstract}

Keywords: quantum information; Shannon entropy; quantum physics; paraconsistent logic; mathematics and computing

\section{Introduction}

The model of the atom was presented by Niels Bohr in 1913, where he proposed that electrons are particles with two kinds of motions in atoms. In the Bohr model, the electrons either move continuously around the nucleus in certain stationary orbits or discontinuously jump between these orbits [1]. Subsequently, with the advances in quantum theory, new concepts, such as the ideas of superposition of states and quantum entanglement, have been proposed. Currently, the physical state of an electron is described by a wave function and in the foundations of quantum mechanics; the wave function is a description of the random discontinuous motion of particles. Moreover, the data on the physical properties of particles are uncertain, and all of the analyses are probabilistic [1,2].

The probability density of the particle appearing in each position is proportional to the square of the modulus of its wave function at every instant. The square of the modulus of the wave function represents not only the probability of a particle being found at a certain location but also the probability of the particle being there [2][3].

In 1925, Heisenberg published results introducing the quantum concepts for particles in matrix analysis. In the matrix formulation, the instantaneous state of a quantum system encodes the probabilities of its measurable properties or "observables," which include energy, position, momentum, and angular momentum. Observables can be either continuous (e.g., the position of a particle) or discrete (e.g., the energy of an electron bound to a hydrogen atom) [2,3].

In 1926, Schrödinger proposed a partial differential equation for the wave functions of particles, such as electrons. The state of a system at a given time is described by a complex wave function, which is also referred to as the state vector in a complex vector space, and this abstract mathematical object enables the calculation of the probabilities of outcomes of concrete experiments $[3,4]$.

Another important consideration is that in quantum mechanics, one can never make simultaneous predictions of conjugate variables, such as position and momentum, to arbitrary precision. In 1927, 
Heisenberg proposed the uncertainty principle, which shows the formal inequality relating the uncertainty of position $\Delta_{x}$ and the uncertainty of momentum $\Delta_{p}$, as follows [3-5]:

$$
\Delta_{x} \Delta_{p} \geq \frac{\mathrm{h}}{2} .
$$

The electrons may be considered (to a certain probability) to be located somewhere within a given region of space. However, their exact positions are unknown. In this condition, contours of constant probability density, which are often referred to as "clouds," may be drawn around the nucleus of an atom to conceptualize where an electron might be located with the most probability [2][5-8]. The probability density is obtained using the square of the amplitude of the wave function, which usually involves a complex quantity. Thus, its value is derived by multiplication with the conjugate complex, as follows [6,7]:

$$
|\psi|^{2}=\psi_{(x, t)}{ }^{*} \psi_{(x, t)} \text {. }
$$

If the wave function is a representative of the sum of probabilities that describe a particle, then it needs to be normalized, as follows [3][8,9]: $\int_{-\infty}^{+\infty} \psi^{*}{ }_{(x, t)} \psi_{(x, t)} d x=1$.

With respect to the logic applied to quantum mechanics among various studies of quantum probabilistic logic formalism, one of the most important was developed by von Neumann in 1932 [11,12]. In his work, von Neumann assumed that each physical system is associated with a Hilbert space $H$ (separable), with its unit vectors corresponding to possible physical states of the system. Each real "observable" random quantity is represented by a self-regulated operator $A$ in $H$, whose spectrum is the set of possible values of $A$ [12]. According to the previous works, mathematics in quantum mechanics can be considered a nonclassical probability calculation, which is supported by a nonclassical propositional logic $[13,14]$.

\subsection{Paraconsistent Logic}

Nonclassical logics are created with the purpose of opposing the binary principles of classical logic, thus providing better conditions for the construction of physical-mathematical models with more approximate results. Currently, there are several types of nonclassical logics, and in general, we can consider that only those logics that are indestructible in the presence of the contradiction are paraconsistent. Therefore, a paraconsistent logic (PL) is a nonclassical logic that has, as its fundamental characteristic, the opposition to the principle of noncontradiction [15-18].

The fundamental theory of PL has been developed in the area of philosophical logic [19], and a formal framework for inconsistent theories was proposed by da Costa [15][17][19]. Further details of the logical formalization of PL, the mathematical implications, and their theorems can be found in [15], [17], and [20].

Blair and Subrahmanian [21] presented applications of PL to logical programming and extended the formalization of three-valued semantics. With this initial work, a theory was developed for possibly inconsistent logic programs using a lattice also known as Belnap's four-valued logic [22], where the set of truth values of four-valued logic is defined as $\tau=\{t, f, \mathrm{~T}, \perp\}$, in which $t, f, \mathrm{~T}$, and $\perp$ are propositions in the language of a program, and they denote true, false, contradictory, and paracomplete, respectively. The set of truth values $\tau$ comprises a complete lattice under the ordering $\leq$, such that $\perp \leq x \geq \mathrm{T}$ for $x \in \tau=\{t, f\}[22,23]$.

\subsection{Paraconsistent Annotated Logic with Annotation of Two Values}

An extended form of PL, the paraconsistent annotated logic (PAL), which has an associated lattice, has been investigated and applied to several fields of science [18] [23,24]. In data analysis systems, the PAL can derive an annotation composed of two degrees of evidence from different sources of information and, in this case, is named paraconsistent annotated logic with annotation of two values-PAL2v [25,26]. The first concepts of PAL2v, which can be applied to artificial intelligence, are presented in [25].

As presented in [18], [26,27], and [28], in the application of PAL2 $v$, the associated lattice is considered an abstract universe $\tau$, where a negation operator allows logical interpretations to result in paraconsistent equations. In the annotation, the first degree of evidence is favorable for the proposition $P$ and is represented by the symbol $\mu$, and the second degree of evidence is unfavorable for the proposition $P$ and is represented by the symbol $\lambda$. These degrees of evidence are normalized, classified as a set of real numbers, and contained in the closed interval $[0,1]$. The annotation assigns a logical state to the proposition $P$. Thus, the information in PAL2v is a paraconsistent logical signal represented by the proposition $P$ with the subscript of the annotation as $(\mu, \lambda): P_{(\mu, \lambda)}$, where the annotation is composed of a pair of the degrees of favorable evidence $(\mu)$ and unfavorable evidence $(\lambda)$.

The paraconsistent symbol $(\mu, \lambda)$ assigns a logical state to proposition $P$ as follows [25,26]: 
1. If the annotation is $(0,1)$, then the degree of favorable evidence is minimum and the degree of unfavorable evidence is maximum, which provides a logical "false" connotation to proposition $P$. This paraconsistent signal defines the logical state "false" $f$.

2. If the annotation is $(1,0)$, then the degree of favorable evidence is maximum and the degree of unfavorable evidence is minimum, which provides a logical "true" connotation to proposition $P$. This paraconsistent signal defines the logical state "true" $t$.

3 . If the annotation is $(1,1)$, then the degree of favorable evidence is maximum and the degree of unfavorable evidence is maximum, which provides a logical true and false connotation to proposition $P$. This paraconsistent signal defines the logical state "inconsistent" $T$.

4. If the annotation is $(0,0)$, then the degree of favorable evidence is minimum and the degree of unfavorable evidence is minimum, which provides a logical false and true connotation to proposition $P$. This paraconsistent signal defines the logical state "paracomplete" $\perp$.

Figure 1(a) shows the lattice FOUR associated with PAL and the representations of the extreme logical states in their vertices through the annotation $(\mu, \lambda)$ of PAL2v [25][29,30].

As discussed in [31,32], and [33], this representation of PAL2v has been recently investigated using an interlaced bilattice also known as the bilattice of Belnap [22,23][34]. A bilattice is a structure $\mathrm{B}=<B, \leq_{t}, \leq_{k}>$, where $\mathrm{B}$ is a nonempty set, and $\left\langle B, \leq_{k}>\right.$ and $<B, \leq_{t}>$ are both bounded lattices, that is, with bottom and top elements. In studies of bilattice, the symbols, $\otimes k$ and $\oplus k$ are used to denote the meet and join operations that correspond to $\leq_{k}$, respectively, and $\otimes t$ and $\bigoplus t$ are used to denote the meet and join operations that correspond to $\leq_{t}$, respectively. The partial order $\leq_{k}$ is intended to represent the knowledge or information order, and $\leq_{t}$ is intended to represent the truth order. In other words, the knowledge order reports on how much information we have about a particular statement $p$, whereas the truth order reports on how confident we are that $p$ is true or false. Interpreting $x \leq_{t} y$, we simply thereby mean that $y$ is truer than $x$; in turn, we interpret $x \leq_{k} y$ to mean that the evidence underlying $x$ is subsumed by the evidence underlying $y$ [22][34].

Figure 1(b) shows the interlaced bilattice of Belnap with the ordering $\leq_{t}$ and $\leq_{k}$ and the representations of the extreme logical states in their four vertices, $t, f$, $\mathrm{T}$, and $\perp$, which denote truth, falsity, both, and none, respectively $[22,23][25]$.

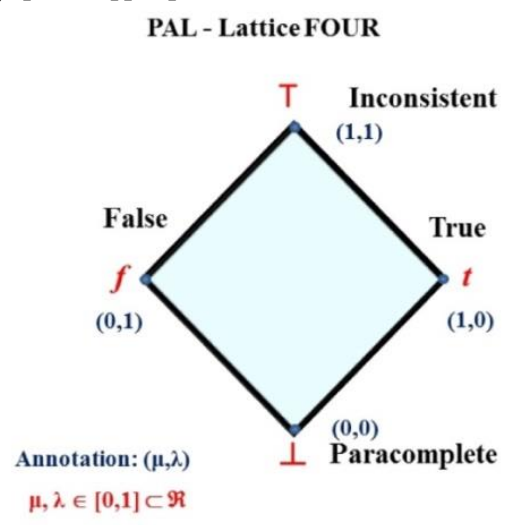

(a)

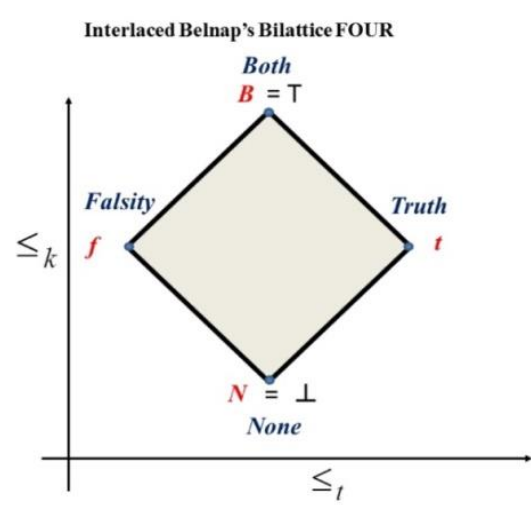

(b)

Fig. 1. Lattices associated with nonclassical logics -LPA and four-valued logic: (a) lattice FOUR associated with PAL2v and representations of the extreme logical states in their vertices through the annotation $(\mu, \lambda) ;(b)$ interlaced bilattice of Belnap with the ordering $s_{t} s_{k}$ and four extreme logical states represented in their vertices.

The paraconsistent equations are obtained from mathematical transformations that map the values arranged in a unitary square on the Cartesian plane (USCP) to the associated bilattice of PAL2v [25,26].

Initially, the degrees of evidence of PAL2v are considered on the USCP (which is also known as lattice $\kappa$ ), from where their values are mapped to lattice FOUR [32]. Given that, in the USCP, the values are allocated to the $x$ - and $y$-axes, the USCP (lattice $\kappa$ ) is mapped to the associated lattice $\tau$ of PAL2v by equating values with the degrees of evidence and implementing the following actions: (a) expansion of $\sqrt{2}$ from the $x$ - and $y$-axes $T_{1}\left(X_{1}, Y_{1}\right)=(x \sqrt{2}, y \sqrt{2}) \quad ; \quad$ (b) $45^{\circ}$ counterclockwise rotation at the origin 
$T_{2}\left(X_{2}, Y_{2}\right)=\left(X_{1} \cdot \cos \theta-Y_{1} \cdot \sin \theta, X_{1} \cdot \sin \theta+Y_{1} \cos \theta\right)$, where $\cos \theta=\frac{1}{\sqrt{2}}$ and $\sin \theta=\frac{1}{\sqrt{2}} ;(\mathrm{c})$ translation of the -1 value from the $y$-axis $T_{3}\left(X_{3}, Y_{3}\right)=\left(X_{2}, Y_{2}-1\right)$ resulting in $T_{3}\left(X_{3}, Y_{3}\right)=(x-y, x+y-1)$.

If $x$ is the value allocated to the $x$-axis of the USCP and $y$ is the value allocated to the $y$-axis of the USCP, then $x=\mu$ and $y=\lambda$. The previously described actions create $T_{1}, T_{2}$, and $T_{3}$ transformations, as described in [26] and [31], which results in the following:

$$
T_{3}\left(X_{3}, Y_{3}\right)=(\mu-\lambda, \mu+\lambda-1) .
$$

We denote the certainty degree $(D c)$ as $X_{3}$ and the contradiction degree $(D c t)$ as $Y_{3}$ [26][31]:

$X_{3}=D c_{(\mu, \lambda)} \rightarrow$ Certainty degree as a function of $\mu$ and $\lambda:$

$$
D c_{(\mu, \lambda)}=\mu-\lambda,
$$

$Y_{3}=\operatorname{Dct}_{(\mu, \lambda)} \rightarrow$ Contradiction degree as a function of $\mu$ and $\lambda:$

$$
\operatorname{Dct}_{(\mu, \lambda)}=\mu+\lambda-1 .
$$

Figure 2(a) shows this mapping with the sequences of actions to obtain the equations of the paraconsistent transformations and associated bilattice of PAL2 $\mathrm{v}$ in the degrees of certainty and contradiction in the $x$ - and $y$-axes.

The maximum negative value of the degree of certainty is -1 at the vertex of the extreme logical state "false" $(f)$ and the maximum positive value is +1 at the vertex of the extreme logical state "true" $(t)$. For these two conditions, the value of the degree of contradiction will always be $\left.0{ }_{\left(D_{c t}(\mu, \lambda)\right.}=0\right)$.

The maximum negative value of the degree of contradiction is -1 at the vertex of the extreme logical state "paracomplete" $(\perp)$, and the maximum positive value is +1 at the vertex of the extreme logical state "inconsistent" (T). For these two conditions, the value of the degree of certainty will always be $0\left(D c_{(\mu, \lambda)}=0\right)$

$[25,26]$. Furthermore, PAL2v, when applied to quantum mechanics, is called paraquantum logic (PqL). In the interlaced $\mathrm{PqL}$ bilattice, the values are represented by a universe of complex numbers, where the degree of contradiction lies in the imaginary axis and the degree of certainty lies in the real axis, with the origin at the point equidistant from the vertices of the bilattice; therefore, in this point, the degrees of certainty and contradiction are both equal to 0 [31-33].

The paraconsistent logical state $\varepsilon_{\tau}$, which defines the paraquantum logical state [31], is considered the point of intersection between the degrees of certainty $\left(D c_{(\mu, \lambda)}\right)$ and contradiction $\left(D c t_{(\mu, \lambda)}\right)$ located in the bilattice FOUR or the PqL bilattice. Therefore, the paraquantum logical state $\varepsilon_{\tau}$ can be expressed as follows $[25,26]:$

$$
\varepsilon_{\tau(\mu, \lambda)}=\left(D c_{(\mu, \lambda)}, D c t_{(\mu, \lambda)}\right) .
$$

Through mapping, the bilattice associated with $\mathrm{PqL}$ becomes a lattice of values, where the equations obtained create pairs of the values of $D c_{(\mu, \lambda)}$ and $D c t(\mu, \lambda)$, which define infinite internal points of intersection. Each internal point of intersection composed of a pair of values is a single paraquantum logical state $\varepsilon_{\tau(\mu, \lambda)}$.

The equations obtained from the transformations enable the determination of the distance between the paraquantum logical state represented by a pair of inseparable values $\left(D c_{(\mu, \lambda)}, D c t_{(\mu, \lambda)}\right)$ and the extreme logical states represented by the vertices of the bilattice. Given that $D c_{(\mu, \lambda)}$ and $D c t_{(\mu, \lambda)}$ are dependent on the $\mu$ and $\lambda$ values, the distance between the logical state resulting from $\varepsilon_{\tau(\mu, \lambda)}$ and one of the extreme logical states $t, f, \mathrm{~T}$, or $\perp$, represented by the vertices of the PqL bilattice, is dependent on the values of $\mu$ and $\lambda$ considered in the physical world. If we know the paraquantum logical state $\varepsilon_{\tau}$ in any region inside the PqL bilattice, then the values of the degrees of evidence can be calculated using the following equations [26][31]:

$$
\mu_{(p)}=\frac{1}{2} D c_{(\mu, \lambda)}+\frac{1}{2} D c t_{(\mu, \lambda)}+\frac{1}{2}
$$

and

$$
\lambda_{(p)}=\frac{-1}{2} D c_{(\mu, \lambda)}+\frac{1}{2} D c t_{(\mu, \lambda)}+\frac{1}{2} .
$$

Non-commutation exists between the degrees of evidence of the $\mathrm{PqL}$ and is explained by the logical negation operation denoted by the symbol $\neg$. The change of position of the degrees of evidence in the 
annotation negates proposition $P$. Therefore, given proposition $P$, its logical negation $\neg P$ is represented by the exchange of the degrees of evidence in the annotation, as follows [31-33]:

$$
\neg(\mu, \lambda)=(\lambda, \mu) \text {. }
$$

An interlaced bilattice [31][33] in addition to the negation operation expressed in Eq.(9) also enables the application of the complementation and conflation operations. The logical complementation operation in the $\mathrm{PqL}$, denoted by the symbol $\lrcorner$, is an explicit complement to the unit of the degrees of evidence in the annotation. Given proposition $P$ and its complement $\lrcorner P$, we can express the complementation operation as follows:

$$
\lrcorner(\mu, \lambda)=(1-\mu, 1-\lambda) .
$$

The logical conflation operation in the PqL, denoted by the symbol $q$, is explained by the negation operation, followed by the complement to the unit of the degrees of evidence in the annotation [31]. Given proposition $P$ and its conflation $\lceil P$, we can express the conflation operation as follows:

$$
\ddagger(\mu, \lambda)=(1-\lambda, 1-\mu) \text {. }
$$

For a logical-mathematical study, the interlaced bilattice associated with PqL can be divided into four quadrants [31]: (a) In Quadrant I, the degrees of certainty and contradiction are positive (there is no operator action on the annotation $(\mu, \lambda)$ ); (b) in Quadrant II, the degree of certainty is negative, while the degree of contradiction is positive (this is an action of the logical negation operator $\neg$ over the annotation $(\mu, \lambda)$ ); (c) in Quadrant III, the degrees of certainty and contradiction are negative (this is an action of the logical complementation operator $\downarrow$ over the annotation $(\mu, \lambda)$ ); and in Quadrant IV, the degree of certainty is positive, while the degree of contradiction is negative (this is an action of the logical conflation operator $\ddagger$ over the annotation $(\mu, \lambda))$.

With the negation, complementation, and conflation operations only over the values of the degrees of certainty and contradiction obtained in Quadrant I, the results of the degrees of certainty and contradiction are obtained in the three other quadrants of the interlaced PqL bilattice. Therefore, given that we detect the paraquantum logical state in Quadrant I with the corresponding values of $D c_{(\mu, \lambda)}$ and $D c t_{(\mu, \lambda)}$, the negation operation results in a negative degree of certainty and an unchanged degree of contradiction. This results in another paraquantum logical state in Quadrant II, which is represented by $\left(-D c_{(\mu, \lambda)},+D c t_{(\mu, \lambda)}\right)[31][33]$. Similarly, the complementation operation on the values of the degrees of certainty and contradiction in Quadrant I results in negative values for both the degrees of certainty and contradiction. This result defines the paraquantum logical state in Quadrant III, which is represented by $\left(-D c_{(\mu, \lambda)},-D c t(\mu, \lambda)\right)$. The conflation operation on the values of the degrees of certainty and contradiction in Quadrant I results in positive values for the degree of certainty and negative values for the degree of contradiction. This result defines the paraquantum logical state in Quadrant IV, which is represented by $\left(+D c_{(\mu, \lambda)},-\operatorname{Dct}_{(\mu, \lambda)}\right)[31,32]$.

Figure 2(b) shows the interlaced PqL bilattice with quadrant operators and paraconsistent equations for reversible logic.

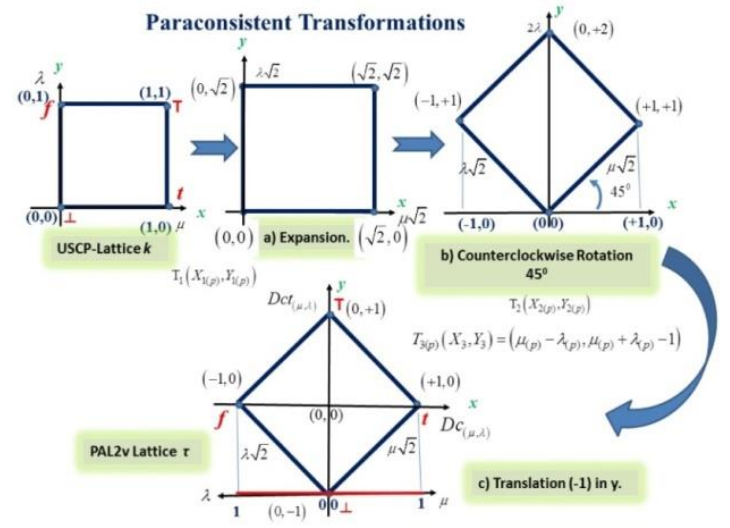

(a)

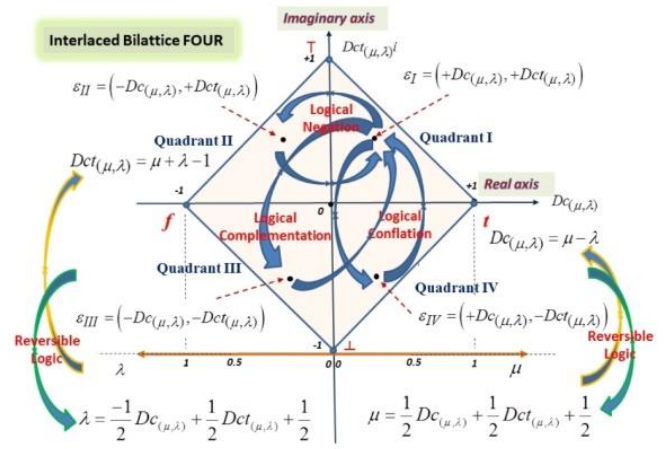

(b)

Fig. 2. Sequences of mapping to obtain the paraconsistent equations and representations in the interlaced $\mathrm{PqL}$ bilattice: (a) sequences followed to obtain the equations of the paraconsistent transformations with counterclockwise rotation; (b) interlaced PqL bilattice with quadrant operators and paraconsistent equations for reversible logic. 


\subsection{Shannon Entropy}

In a work by Shannon in 1948 [35], the basis of the mathematical theory of communication, or information theory, was established. Moreover, Shannon's work emphasizes a fundamental concept, that is, the entropy of the information, which has become well known as the Shannon entropy $H_{(\mathrm{s})}$. The Shannon entropy has complementary interpretations that can be either information quantity (after measurement) or uncertainty (before measurement) in a given probability distribution [36,37]. To establish the current concept that $H_{(\mathrm{s})}$ is a function of entropy, similar to Boltzmann's $H$ theorem, Shannon defined some statistical concepts through the equation $H_{(s)}=-k \sum_{i=1}^{n} p i \log p i$, where $p i$ is the probability of a system being in cell $i$ of its phase space, and $k$ corresponds only to a certain unit of measure [36][38,39].

The equation of entropy in the case of two variables, that is, $p$ and $q$ (where $q=1-p$ ), is written as follows:

$$
H_{(s)}=-k[p \log p+q \log q],
$$

where $p$ is the probability, $q$ is its complement $(1-p)$, and the constant $k$ depends on the variable used [36, 37]. As can be seen in [36] and [37], to obtain the maximum unitary value of $H_{(s)}$ in Eq. (12), $k$ is calculated as $k=\frac{\log 10}{\log 2}=\frac{1}{\log 2}=\frac{1}{0.301029995} ; 3.321928$.

\subsection{Rydberg's Formula}

In 1890, before Bohr introduced his model of the atom, Johannes Rydberg developed formulas describing the wavelengths or frequencies of light in various series of related spectral lines [40]. Later, Bohr expressing results in terms of wavenumber, not wavelength, combined these formulas. These studies resulted in the following equation [1][40]:

$$
\frac{1}{\lambda}=R_{\infty} Z^{2}\left(\frac{1}{n_{1}^{2}}-\frac{1}{n_{1}^{2}}\right)
$$

where

$\lambda$ is the wavelength of the photon (wavenumber $=1 /$ wavelength);

$Z$ is the atomic number of the atom;

$n_{1}$ is the principal quantum number of an energy level, for the atomic electron transition;

$n_{2}$ is the principal quantum number of an energy level for the atomic electron transition, with $n_{2}>n_{1}$; and

$R_{\infty}$ is the Rydberg's constant, calculated as $R_{\infty}=\frac{m_{e} e^{4}}{8 \epsilon_{0}{ }^{2} h^{3} c}=1.0973731568539 \mathrm{~m}^{-1}$,

where $m_{e}$ is the mass of the electron, $e$ is the elementary charge of the electron, $\in_{0}$ is the permittivity of free space, $c$ is the speed of light, and $h$ is the Planck's constant.

\subsection{Bernoulli Distribution}

The probability $p$ is an outcome that generates the degrees of evidence for the analysis of proposition $P$ for affirmation (true) or refutation (false). One form of representation whose results can be applied to the interlaced PqL bilattice is the Bernoulli trial process [31][41]. For this representation, we derive the random distribution of variable $X$, such that [31] $\operatorname{Pr}(X=1)=p$ and $\operatorname{Pr}(X=0)=q$. The expectation value is calculated using $\rightarrow E(X)=p$, and the variance of $X$ is written as $\operatorname{Var}(X)$. The variance is a measure of how much the value of $X$ varies from the expectation $E(X)$ and is defined as $\operatorname{Var}(\mathrm{X})=p-p^{2}$.

The standard deviation of the probability distribution is denoted by the symbol $\sigma$ and is defined as the square root of the variance $\operatorname{Var}(X)$ :

$$
\sigma=\sqrt{\operatorname{Var}(X)} .
$$


A graph of $\operatorname{Var}(X)$ as a function of $p \in[0,1]$ exhibits a parabola that opens downward [31][41].

The paraconsistent model of the atom will be presented and analyzed in this paper. We compare the use of probability in the Shannon entropy function, which will form the degrees of evidence, and the use of Bernoulli distribution to determine the probability value $p$ of the paraconsistent analysis.

In the first stage of the analysis, we show the trajectory of the logical states in the ground state and its main equations obtained in Quadrant I of the interlaced PqL bilattice. Moreover, the negation, complementation, and conflation operations are applied, and the model of the complete atom in the $x y$ plane is formed in the perception of an observer in the vector base X. In the second stage of the analysis, the modeling equations of the complete atom are presented, and the trajectories of degenerate and nondegenerate quantum states are highlighted. In the third stage of the analysis, the modeling equations of the energy layers are derived from the mapping of the degrees of evidence that differs in terms of the direction of rotation, which is now done clockwise. In this manner, the paraconsistent model of an atom in the $x y$ plane is formed in the perception of an observer in the vector base Y. Finally, the results of an example of the application of the paraconsistent model of an atom are correlated with the energy values extracted from the Rydberg formulas and presented based on the hydrogen atom. The results of the hydrogen atom show the curves obtained from the analysis of signals using only Quadrant I of the interlaced PqL bilattice. With this final model is presented a method of using Raman spectroscopy signals for the detection of lubricating mineral oil quality.

\section{Materials and Methods}

In the construction of the paraconsistent model of the atom, the concepts and equations of $\mathrm{PqL}$ and the logarithmic function of the Shannon entropy $H_{(\mathrm{s})}$ are used. These fundamentals, equations, and concepts are applied to the in-depth analysis of the interlaced bilattice associated with PqL. In the proposed model, to represent the probabilistic functions according to the fundamentals of $\mathrm{PqL}$, it is necessary to establish state vectors with unitary modules and that define the orbital paths and energy layers of the atom.

\subsection{State Vectors and Internal and External Orbit Trajectories of Paraquantum Logical States}

We present below the representation of the paraquantum logical states related to the unitary module state vectors that are installed in the interlaced bilattice.

\subsubsection{Pyint-Internal State Vector}

We consider an internal state vector with a unitary module $(\mathrm{P} \psi$ int $)$ and that has its origin located at the vertex of the true logical state $(t)$ of the interlaced PqL bilattice. In this point, $D c_{(\mu, \lambda)}=+1$ and $D c t_{(\mu, \lambda)}=0$. Therefore, if the paraquantum logical state of the origin is expressed as $\varepsilon_{\tau}=\left(D c_{(\mu, \lambda)}, D c t_{(\mu, \lambda)}\right)=(+1,0)$, then the variation of the inclination angle of the vector $(\mathrm{P} \psi \mathrm{int})$ in Quadrant I will be $0 \leq \alpha_{\psi} \leq \frac{\pi}{4}$ in radians. Given that the modulus is unitary, the variation of the inclination angle $\alpha_{\psi}$ of the vector $\mathrm{P} \psi$ int will occur in the interlaced $\mathrm{PqL}$ bilattice, with a curvilinear trajectory defined by the paraquantum logical states located at its end.

The internal trajectory in Quadrant I of the interlaced PqL bilattice is defined in Eq. (6).

\subsubsection{P $\psi_{\text {ext }}$-External State Vector}

For an external state vector with a unitary module $\left(\mathrm{P} \psi_{\mathrm{ext}}\right)$ and that has its origin at the point equidistant from the vertices of the interlaced PqL bilattice $\varepsilon_{\tau}=\left(D c_{(\mu, \lambda)}, D c t_{(\mu, \lambda)}\right)=(0,0)$, the equation of the paraquantum logical states that form the external trajectory in Quadrant I can be described as a function of the value of $\alpha_{\psi}$ and can be expressed as follows: 


$$
\varepsilon_{\psi(\alpha \psi)} I=(1-\cos (\alpha \psi), \operatorname{sen}(\alpha \psi)) .
$$

This expression is similar to the following function:

$$
\varepsilon_{(\mu, \lambda)} I=\left(1-D c_{(\mu, \lambda)}, D c t_{(\mu, \lambda)}\right),
$$

where $\mu$ and $\lambda$ are the degrees of evidence.

\subsubsection{P $\psi_{\text {Cext }}$-Complementary External State Vector}

The external trajectory of the paraquantum logical states in Quadrant I can be completed for the variation of the inclination angle $\alpha_{\psi}$ of another vector, that is, $\mathrm{P} \psi_{\text {Cext. }}$ With the same values of $\mu$ and $\lambda$, an external complementary vector $\left(\mathrm{P} \psi_{\text {CextII }}\right)$ with a unitary module is created simultaneously, with its origin at the point $\varepsilon_{\tau}=\left(D c_{(\mu, \lambda)}, D c t_{(\mu, \lambda)}\right)=(0,0)$ and its angle of inclination having a variation of $\frac{\pi}{4} \leq \alpha_{\psi} \leq \frac{\pi}{2}$ in radians. With the same quantitative values of the degrees of evidence, this complementary operation is applied to the degrees of certainty and contradiction, generating the paraquantum logical state $\varepsilon_{(\mu, \lambda)}=\psi_{I 2(P q L) E 1}$.

The generated paraquantum logical state establishes the orbital trajectory of the state vector $\mathrm{P} \psi_{\mathrm{I} 2}$, whose inclination presents a variation of $45^{\circ}$ to $90^{\circ}$, that is, an angular variation of $\frac{\pi}{4}$ to $\frac{\pi}{2}$ radians.

The paraquantum logical state, which features the orbital trajectory at the end of the new complementary state vector, with the completed action in the degrees of certainty and contradiction, is represented by $\varepsilon_{(\mu, \lambda)} I I=\uparrow \varepsilon_{(\mu, \lambda)} I$ in the following expression:

$$
\varepsilon_{(\mu, \lambda)} I I=\left(\left(1-D c t_{(\mu, \lambda)}\right), 1-\left(1-D c_{(\mu, \lambda)}\right)\right) .
$$

In Quadrant I of the interlaced PqL bilattice, $\mu$ and $\lambda$ are represented by probabilistic functions $\mu_{(p)}$ and $\lambda_{(p)}$, which must present results that have their values varying simultaneously in the corresponding intervals, that is, $0.5 \leq \mu_{(p)} \leq 1.0$ and $0.5 \leq \lambda_{(p)} \leq 1.0$, respectively.

Figure 3 shows Quadrant I of the interlaced $\mathrm{PqL}$ bilattice with the state vector with a unitary module, which, with the variation of the inclination angle $\alpha_{\psi}$, establishes the internal trajectory of the paraquantum logical states. In the same mode, the external state vectors $\mathrm{P} \psi_{\text {extI }}$ and $\mathrm{P} \psi_{\text {CextI }}$ establish the complete external trajectory of Quadrant I.

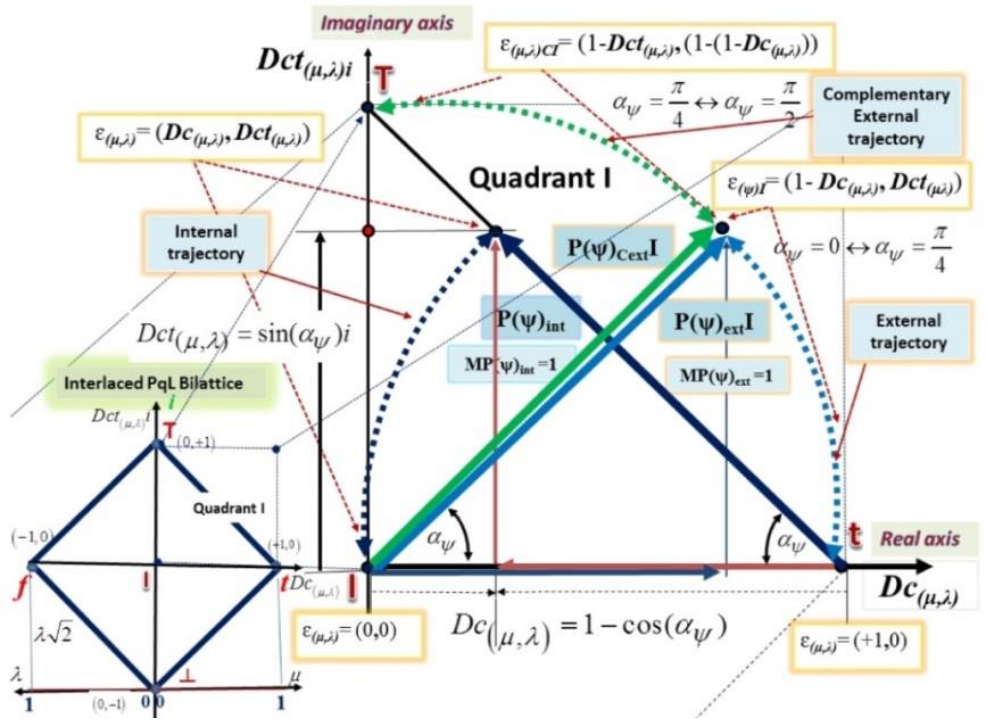

Fig. 3. Quadrant I of the interlaced PqL bilattice, with the internal state vector $(\mathrm{P} \psi$ int $)$ and two external state vectors $\left(\mathrm{P} \psi_{\text {extI }}\right.$ and $\left.\mathrm{P} \psi_{\text {CextI }}\right)$ that establish the internal and external orbit trajectories of the paraquantum logical state $\varepsilon_{(\mu, \lambda)}$.

\subsection{Representation of the Degrees of Evidence of PqL as Probabilistic Functions}

First, we consider that $\mu_{(p)}$ is a probabilistic function $f_{(p)}=X$, such that $p \in \Re$ and it is contained in the closed interval $[0,1]$. Therefore, $p$ represents a probability value, and the normalized values $X$ and $X^{\prime}$ should be adapted to the interlaced PqL bilattice. The two probability values must also be presented to form an annotation. We also consider that as an initial condition, the two probabilistic sources 1 and 2 are out of 
phase at the angle $\Theta$, such that in the amplitude variation of the probability value $p$, the probabilistic function of source 2 generates another function; that is, $\lambda_{(p)}=X^{\prime}$. These two probabilistic functions must have the following characteristics: (a) when $X$ is at its maximum unitary value, that is, $\mu_{(p)}=X=1$, the difference between $X$ and $X^{\prime}$ will be equal to $\Delta_{(X p)}=\left(X-X^{\prime}\right) \rightarrow_{\Delta_{(X p)}}=\left(1-\frac{1}{\sqrt{2}}\right)$; (b) when $X$ is at half its maximum value, that is, $\mu_{(p)}=X=\frac{1}{2}$, the difference between $X$ and $X^{\prime}$ will be null $\Delta_{(X p)}=X-X^{\prime}=\left(\frac{1}{2}-\frac{1}{2}\right)=0$. From the reference probability value at source 1 , which is considered a degree of favorable evidence $\mu_{(p)}$, the degree of unfavorable evidence $\lambda_{(p)}$ under the previously mentioned conditions is derived as follows:

$$
\lambda_{(p)}=\sqrt{\frac{\mu_{(p)}}{2}} .
$$

From Eq. (4), the degree of certainty of the interlaced PqL bilattice, which is now a probabilistic function, can be calculated as follows:

$$
D c_{(p)}=\mu_{(p)}-\sqrt{\frac{\mu_{(p)}}{2}} .
$$

In the same manner, the degree of contradiction shown in Eq. (5) is also a probabilistic function, which can be calculated as follows:

$$
D t_{(p)}=\mu_{(p)}+\sqrt{\frac{\mu_{(p)}}{2}}-1 .
$$

From Eq. (6), the paraquantum logical state $\psi_{\mathrm{PqL}}$ that appears in the interlaced $\mathrm{PqL}$ bilattice will be represented by two probabilistic functions, as follows:

$$
\psi \tau_{(p)}=\left(D c_{(p)}, D c t_{(p)}\right) .
$$

In the representation of the functions, the paraquantum logical state $\psi \tau_{(p)}$ will form a probabilistic trajectory into the interlaced $\mathrm{PqL}$ bilattice. Moreover, the paraquantum logical state $\psi_{\mathrm{PqL}}$ will form a probabilistic trajectory out of the interlaced $\mathrm{PqL}$ bilattice, with the origin of the state vector at the point equidistant from the vertices, thereby located where $D c_{(p)}=0$ and $D c t(p)=0$. In this case, the paraquantum logical state $\psi_{\mathrm{p}}$ that forms an external orbit trajectory will be constructed with two probabilistic functions, as follows:

$$
\psi_{(p)}=\left(1-D c_{(p)}, D c t_{(p)}\right),
$$

where $D c_{(p)}$ is obtained using Eq. (19), and $D c t(p)$ is obtained using Eq. (20).

\subsection{Representation of Fundamental PqL-Equations}

In this work, we will construct a paraconsistent model of the atom using the Shannon entropy to operate as a probabilistic function representative of the degrees of evidence $(\mu, \lambda)$ in the interlaced PqL bilattice. In this manner, the fundamental PqL-equations as degree of evidence equations will be probabilistic functions that will be inserted in the energy equations of the paraconsistent model of the atom.

\subsubsection{Shannon Normalization Factor}

To apply the Shannon entropy function to the $\mathrm{PqL}$ equations, we will introduce an adjustment dimensionless value represented by the symbol 1, which will be called the Shannon normalization factor. The 1 value is calculated as follows:

Being that the Shannon entropy from Eq. (12) is represented by the probabilistic logarithmic function $H_{(s)}=-k[p \log p+q \log q]$, the maximum unitary value of $H_{(s)}$ can be obtained when $k=\frac{1}{\log 2}$; then we will 
make a representation of $k$ in which the equality of constants is satisfied as $k=\frac{1}{\log 2}=1 \pi$. Thus, the Shannon normalization factor value 1 is obtained by the equation: $1=\frac{1}{\pi \log 2}$, where $\pi=3.14159265358$, that results at $1=1.057402554$.

\subsubsection{Degrees of Evidence as Shannon Entropy Functions}

With these dimensionless values relationships, the function of Shannon entropy for application in PqL, which we named $H_{(s) P q L}$, can be represented in a normalized mode as follows:

$$
H_{(s) P q L}=-1 \pi[p \log p+q \log q],
$$

where $\pi$ is the constant of value $(\pi=3.14159265358 \ldots), p$ is the probability value,

$q$ is the complement of the probability value $[q=(1-p)]$, and

1 is the Shannon normalization factor extracted from $k$, with $1=\frac{1}{\pi \log 2} ; 1.057402554$.

The variation of the resulting values of the function $H_{(s) P q L}$ is expressed in the range $0 \leq H_{(s) P q L} \leq 1$; therefore, the Shannon's normalized entropy has the variation values contained within the same range as that established for the PqL degrees of evidence.

For the paraconsistent model of the atom, the probabilistic function of the degree of favorable evidence of the $\mathrm{PqL} \mu_{(P q L)}$ can be expressed as follows:

$$
\mu_{(P q L)}=H_{(s) P q L},
$$

where $H_{(s) P q L}$ is the Shannon entropy function presented in Eq. (23).

According to Eq. (18), with the inclusion of the Shannon entropy, the probabilistic function of the degree of unfavorable evidence can be expressed as follows:

$$
\lambda_{(P q L)}=\sqrt{\frac{H_{(s) P q L}}{2}},
$$

where $H_{(s) P q L}$ is the probabilistic Shannon entropy function presented in Eq. (23).

With these two last equations, $H_{(s) P q L}=1$ means high entropy. In this condition, the degree of favorable evidence of the PqL (Eq. 24), will be of unit value $\mu_{(P q L)}=1$, and the degree of unfavorable evidence (Eq. $25)$, will be $\lambda_{(P q L)}=\frac{\sqrt{2}}{2}$. Likewise, $H_{(s) P q L}=0.5$ means low entropy. In this condition the degree of favorable evidence, by Eq. (24), will be $\mu_{(P q L)}=0.5$, and the degree of unfavorable evidence, by Eq. (25), will be $\lambda_{(P q L)}=0.5$.

\subsection{PqL Energy Equations}

The degrees of evidence with their probabilistic representations created by the Shannon entropy function $H_{(s) P q L}$ are used in the equations to obtain the degrees of certainty and contradiction resulting in dimensionless values contained in the range $[-1,+1]$. Since the PqL equations are functions that deal with normalized dimensionless values between 0 and 1 , when multiplied by a known maximum energy value, they will represent the energy value in each condition expressed by the paraquantum logical states. Thus, in the paraconsistent model of an atom, we can consider that these $\mathrm{PqL}$ equations involve quantized energies through the values established by the unit modulus vectors $\mathrm{P} \psi_{\text {int }}, \mathrm{P} \psi_{\text {ext }}$, and $\mathrm{P} \psi_{\text {CextII. In this condition, at the }}$ ground-state level of the atom, the degrees of certainty $D c_{(P q L)}$ and contradiction $D c_{(P q L)}$ represent the energy values, which are obtained using the quantized probabilistic evidence degrees.

From Eq. (19), the probabilistic certainty degree of the ground state (level $E_{1}$ ) can be calculated as follows:

$$
D c_{(P q L) E 1}=H_{(s) P q L}-\sqrt{\frac{H_{(s) P q L}}{2}} .
$$


From Eq. (20), the probabilistic contradiction degree of the ground state (level $E_{1}$ ) can be calculated as follows:

$$
\operatorname{Dct}_{(P q L) E 1}=H_{(s) P q L}+\sqrt{\frac{H_{(s) P q L}}{2}}-1 \text {. }
$$

The values of the degrees of certainty and contradiction considered in the set of complex numbers $£$, with their quantized probabilistic functions, represent the energy of the atom. In the proposed paraconsistent model of an atom, the ground state (level $E_{1}$ ) is represented by the point of origin of the real and imaginary axes, which will be located at the point equidistant from the vertices of the interlaced PqL bilattice. In this representation, the paraconsistent logical state $\psi_{P q l}$ that defines the external orbital trajectory in the ground state, representing the complex numbers in Quadrant I, is expressed as follows:

$$
\psi_{I 1(P q L) E 1}=\left(1-D c_{(P q L) E 1}, D c t(P q L) E 1^{i}\right) .
$$

The probabilistic functions of the paraquantum logical state $\psi_{I(P q L) E 1}$ establish the ground state (level $\left.E_{1}\right)$ and the external orbital trajectory in the ground state of the model of an atom at the end of the state vector $\mathrm{P} \psi_{\mathrm{I} 1}$ with a unitary module; that is, $M \psi_{E 1}=\sqrt{\left(1-D c_{(P q L) E 1}\right)^{2}+(D c t(P q L) E 1)^{2}}$ or

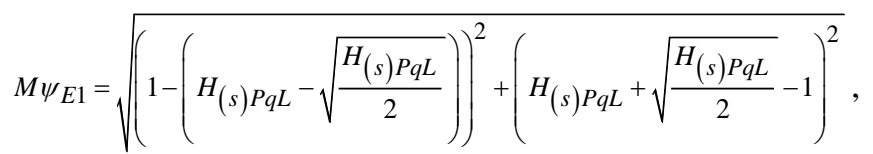

where $H_{(s) P q L}$ is the probabilistic Shannon entropy function presented in Eq. (23).

\subsection{Analogies between Quantum Mechanics and Paraquantum Logic}

With these logical-mathematical considerations, some concepts of PqL can be compared with the concepts of quantum mechanics on the basis of the equations obtained in Quadrant I of the interlaced $\mathrm{PqL}$ bilattice. Therefore, in quantum mechanics, the quantum state is represented by $|\psi\rangle=\alpha|0\rangle+\beta|1\rangle$ and the vector norm is represented by $\||\psi\rangle \|=|\alpha|^{2}+|\beta|^{2}$. The same paraquantum logical state in quantum mechanics will be achieved, with the following relations of equality: $\alpha=\left(1-D c_{(P q L)}\right)$ and $\beta=D c t(P q L)$. The quantum state of the quantum mechanics in the $\mathrm{PqL}$ is represented by the following well-known Dirac notation:

$$
|\psi\rangle=\left(1-D c_{(P q L)}\right)|0\rangle+D c t_{(P q L)}|1\rangle .
$$

In general, for $n$ number of states related to En layers of energies:

$$
\left|\psi_{n}\right\rangle=\left(1-D c_{(P q L) E n}\right)|0\rangle+D c t_{(P q L) E n}|1\rangle .
$$

The representation of the degrees of certainty and contradiction and the ground state in level $E_{1}$ will be unitary $\left(E_{1}=1\right)$ and is represented by

$$
E_{1 \text { Total }}=\left(1-\left(H_{(s) P q L}-\sqrt{\frac{H_{(s) P q L}}{2}}\right)\right)^{2}+\left(\left(H_{(s) P q L}+\sqrt{\frac{H_{(s) P q L}}{2}}\right)-1\right)^{2},
$$


where the potential energy of the ground state is $E_{P 1}=\left(1-\left(H_{(s) P q L}-\sqrt{\frac{H_{(s) P q L}}{2}}\right)\right)^{2}$, and the kinetic energy of the ground state is $E_{c 1}=\left(\left(H_{(s) P q L}+\sqrt{\frac{H_{(s) P q L}}{2}}\right)-1\right)^{2}$ and $H_{(s) P q L}$ is the probabilistic Shannon entropy function presented in Eq. (23).

\subsection{PqL Energy Equations for the Observer in the Vector Base X}

In Quadrant I of the interlaced PqL bilattice, the Shannon entropy functions simultaneously create the trajectories of the paraquantum logical states at the ends of two state vectors, thus establishing the ground state (level $E_{1}$ ) of the quantum state of the particle. The state vector P $\psi_{\text {CextI }}$ constructed with the complementary action, in relation to the original vector $\mathrm{P} \psi_{\text {extI }}$, has the same characteristics and differs only in terms of the angular variation. For the $X$ observer, as defined in the mapping shown in Fig. 3, the projections of the real values in the $x$-axis, which represent the potential energy, and the imaginary values in the $y$-axis, which represent the kinetic energy, vary proportionally, indicating the equilibrium of values against the inherent probabilistic uncertainties of quantum mechanics.

\subsubsection{Ground State of the Atom in the Paraconsistent Model}

The energies of the ground state are represented by the $\mathrm{PqL}$ equations, with the adapted function of the Shannon entropy having only the probability $p$ as its variable.

Using the logical operations of negation, complementation, and conflation, as well as the fundamentals of $\mathrm{PqL}$, we will now define the $n$ energy equations that form the $n$ layers of the paraconsistent model of the atom.

Initially, through these operations, the ground-state energy equations of the three other quadrants of the interlaced $\mathrm{PqL}$ bilattice are obtained.

The negation operator applied to the functions of the paraquantum logical states that mark the orbital trajectory of the particle in the ground state of Quadrant I produces Quadrant II, as follows:

$$
\begin{gathered}
\neg \psi_{(I) 1}=\psi_{(I I) 1}=(-\alpha, \beta) \rightarrow \psi_{(I I) 1}=\left(D c_{(P q L) E n}-1, D c t(P q L) E n\right), \\
\neg \psi_{(I) 2}=\psi_{(I I) 2}=(\beta-1, \alpha-1) \rightarrow \psi_{(I I) 2}=\left(D c t,(P q L) E n-1,-D c_{(P q L) E n}\right) .
\end{gathered}
$$

In Quadrant III, the complementation operator applied to the functions of the paraquantum logical states that mark the orbital trajectory of the particle in the ground state of Quadrant I produces the following expressions:

$$
\begin{aligned}
& \downarrow \psi_{(I) 1}=\psi_{(I I I) 1}=(-\alpha,-\beta) \rightarrow \psi_{(I I I) 1}=\left(D c_{(P q L) E n}-1,-D t_{(P q L) E n}\right), \\
& \downarrow \psi_{(I) 2}=\psi_{(I I I) 2}=(\beta-1,1-\alpha) \rightarrow \psi_{(I I) 2}=\left(D t_{(P q L) E n}-1,1-\left(1-D c_{(P q L) E n}\right)\right) .
\end{aligned}
$$

In Quadrant IV, the conflation operator applied to the functions of the paraquantum logical states that mark the orbital trajectory of the particle in the ground state of Quadrant I produces the following expressions:

$$
\begin{aligned}
& +\psi_{(I) 1}=\psi_{(I V) 1}=(\alpha,-\beta) \rightarrow \psi_{(I V) 1}=\left(1-D c_{(P q L) E n},-D c t(P q L) E n\right) \\
& +\psi_{(I) 2}=\psi_{(I V) 2}=(\beta, 1-\alpha) \rightarrow \psi_{(I V) 2}=\left(1-D c t_{(P q L) E n}, D c_{(P q L) E n}\right) .
\end{aligned}
$$


These PqL logical operations create the paraconsistent model of the atom, where the probabilistic trajectory of the particle in the ground state is a unit-radius circle composed of the Shannon entropy functions introduced in the degrees of certainty and contradiction equations. These probabilistic trajectories related to the ground state are shown in the graphics of the results section.

\subsection{Energy Layers of Degenerate and Nondegenerate States}

In this work, we consider that the layers of the atom that relate to the degenerate states are represented by the energy that is related to the fundamentally pure state but is not aligned to the $x$-axis of the real values. In the interlaced $\mathrm{PqL}$ bilattice, the degenerate states have different values of contradiction degrees, which bring them close to the extreme logical state of inconsistency in Quadrants I and II and the extreme logical state of paracompleteness in Quadrants III and IV.

In the same manner, the layers of the atom that relate to the nondegenerate states are represented by the energy that is related to the fundamentally pure state. In the interlaced PqL bilattice, the nondegenerate states are aligned to the $x$-axis of the real values and thus to the axis of the degrees of certainty. The nondegenerate states have the same values of contradiction degrees and different values of certainty degrees, which bring them close to the extreme logical state of true $(t)$ in Quadrants I and IV and the extreme logical state of false (f) in Quadrants II and III.

\subsubsection{Second Layer of Energy}

With these considerations, for the second layer, the degree of favorable evidence $\mu$ is expressed in Eq. (24), and that of unfavorable evidence is expressed in Eq. (25).

We can maintain a constant difference between the two degrees of evidence within a reasonable range of the probability variation $p$. For this, the degree of unfavorable evidence can be obtained by multiplication with the degree of favorable evidence, such that $\lambda_{(P q L) E E 2}=\mu_{(P q L) E 2} \times \lambda_{(P q L) E 2}$ or

$$
\lambda_{(P q L) E E 2}=H_{(s) P q L} \sqrt{\frac{H_{(s) P q L}}{2}} .
$$

With these values of the degrees of evidence, the degree of certainty for the energy level $E_{2}$ will have a constant value over a reasonable range of probability variation $p$. Therefore, $D c_{(P q L) E 2}=\mu_{(P q L) E 2}-\lambda_{(P q L) E E 2}$

or

$$
D c_{(P q L) E 2}=H_{(s) P q L}-\left(H_{(s) P q L} \sqrt{\frac{H_{(s) P q L}}{2}}\right) .
$$

Moreover, the degree of contradiction for the energy level $E_{2}$ can be derived as follows:

$$
\operatorname{Dct}_{(P q L) E 2}=H_{(s) P q L}+\left(H_{(s) P q L} \sqrt{\frac{H_{(s) P q L}}{2}}\right)-1,
$$

where $H_{(s) P q L}$ is the Shannon entropy function presented in Eq. (23).

In the second layer, the degenerate paraquantum logical state will be represented by the function:

$$
\psi_{(P q L) E 2}=\left(1-D c_{(P q L) E 2}, D c t(P q L) E 2\right)
$$

or by the Dirac notation for the $X$ observer:

$$
|\psi\rangle_{(P q L) E 2}=\left(1-D c_{(P q L) E 2}\right)|0\rangle+D c t_{(P q L) E 2}|1\rangle,
$$

where $D c_{(P q L) E 2}$ is presented in Eq. (34), and $D c t_{(P q L) E 2}$ is presented in Eq. (35). 
Given the relation to the pure state of the ground state, in the second layer, the pure or nondegenerate paraquantum logical state will be represented by the complement expressed in Eq. (34) and the function expressed in Eq. (27), as follows:

$$
\psi_{(P q L) E 2 \text { pure }}=\left(1-D c_{(P q L) E 2}, D c t(P q L) E 1\right) .
$$

\subsubsection{Third Layer of Energy}

In the third layer, the degree of favorable evidence $\mu$ is equal to the degree of unfavorable evidence previously presented in Eq. (25). Therefore, $\mu_{(P q L) E 3}=\lambda_{(P q L) E 2}$ or

$$
\mu_{(P q L) E 3}=\sqrt{\frac{H_{(s) P q L}}{2}} .
$$

In this manner, the intermediary degree of unfavorable evidence will be obtained through the square root of $\mu_{(P q L) E 3}$. Therefore,

$$
\lambda_{(P q L) E 3}=\sqrt{\sqrt{\frac{H_{(s) P q L}}{2}}} .
$$

The degree of unfavorable evidence of the third layer will be obtained by multiplication with the degree of favorable evidence, such that $\lambda_{(P q L) E E 3}=\mu_{(P q L) E 3} \times \lambda_{(P q L) E 3}$ or

$$
\lambda_{(P q L) E E 3}=\sqrt{\frac{H_{(s) P q L}}{2}} \times \sqrt{\sqrt{\frac{H_{(s) P q L}}{2}} .}
$$

With these values of the degrees of evidence, the degree of certainty for the energy level $E_{3}$ will have a constant value over a reasonable range of probability variation $p$. Therefore, the degree of certainty for the third layer of energy will be computed using $D c_{(P q L) E 3}=\mu_{(P q L) E 3}-\lambda_{(P q L) E E 3}$ or

$$
D c_{(P q L) E 3}=\sqrt{\frac{H_{(s) P q L}}{2}}-\left(\sqrt{\frac{H_{(s) P q L}}{2}} \times \sqrt{\sqrt{\frac{H_{(s) P q L}}{2}}}\right) .
$$

Moreover, the degree of contradiction for the energy level $E_{3}$ can be derived as follows:

$$
\operatorname{Dct}_{(P q L) E 3}=\sqrt{\frac{H_{(s) P q L}}{2}}+\left(\sqrt{\frac{H_{(s) P q L}}{2}} \times \sqrt{\sqrt{\frac{H_{(s) P q L}}{2}}}\right)-1 .
$$

In the third layer, the degenerate paraquantum logical state will be represented by the function:

$$
\psi_{(P q L) E 3}=\left(1-D c_{(P q L) E 3}, D c t(P q L) E 3\right)
$$

or

$$
|\psi\rangle_{(P q L) E 3}=\left(1-D c_{(P q L) E 3}\right)|0\rangle+\operatorname{Dct}_{(P q L) E 3}|1\rangle,
$$

where $D c_{(P q L) E 3}$ is presented in Eq. (42), and $D c t_{(P q L) E 3}$ is presented in Eq. (43).

In the third layer, the pure or nondegenerate paraquantum logical state will be represented by the functions expressed in Eqs. (42) and (27), as follows:

$$
\psi_{(P q L) E 3 \text { pure }}=\left(1-D c_{(P q L) E 3}, D c t_{(P q L) E 1}\right) .
$$

\subsubsection{Fourth Layer of Energy}

In the fourth layer, the degree of favorable evidence is equal to the degree of unfavorable evidence previously derived. Therefore, $\mu_{(P q L) E 4}=\lambda_{(P q L) E 3}$ or

$$
\mu_{(P q L) E 4}=\sqrt{\sqrt{\frac{H_{(s) P q L}}{2}}} .
$$

The degree of unfavorable evidence will be obtained through the square root of $\mu_{(P q L) E 4}$. Therefore, 


$$
\lambda_{(P q L) E 4}=\sqrt{\sqrt{\sqrt{\frac{H_{(s) P q L}}{2}}}} .
$$

The degree of unfavorable evidence of the energy level $\left(E_{4}\right)$ of the current state is calculated by multiplication, such that $\lambda_{(P q L) E E 4}=\mu_{(P q L) E 4} \times \lambda_{(P q L) E 4}$ or

$$
\lambda_{(P q L) E E 4}=\sqrt{\sqrt{\frac{H_{(s) P q L}}{2}}} \times \sqrt{\sqrt{\sqrt{\frac{H_{(s) P q L}}{2}}} .}
$$

The degree of certainty for energy level $E_{4}$ can be derived as follows:

$$
D c_{(P q L) E 4}=\sqrt{\sqrt{\frac{H_{(s) P q L}}{2}}}-\sqrt{\sqrt{\frac{H_{(s) P q L}}{2}}} \times \sqrt{\sqrt{\sqrt{\frac{H_{(s) P q L}}{2}}} .}
$$

The degree of contradiction for energy level $E_{4}$ can be derived as follows:

$$
\operatorname{Dct}_{(P q L) E 4}=\sqrt{\sqrt{\frac{H_{(s) P q L}}{2}}}+\sqrt{\sqrt{\frac{H_{(s) P q L}}{2}}} \times \sqrt{\sqrt{\sqrt{\frac{H_{(s) P q L}}{2}}}}-1 .
$$

In the fourth layer, the degenerate paraquantum logical state will be represented by the function:

$$
\psi_{(P q L) E 4}=\left(1-D c_{(P q L) E 4}, D c t(P q L) E 4\right)
$$

or

$$
|\psi\rangle=\left(\left(1-D c_{n d(P q L) E 4}\right)\right)|0\rangle+\left(D c t_{n d(P q L) E 4}\right)|1\rangle,
$$

where $D c_{(P q L) E 4}$ is presented in Eq. (50), and $D c t(P q L) E 4$ is presented in Eq. (51).

In the fourth layer, the pure or nondegenerate paraquantum logical state will be represented by the functions expressed in Eqs. (50) and (27), as follows:

$$
\psi_{(P q L) E 3 \text { pure }}=\left(1-D c_{(P q L) E 4}, D c t(P q L) E 1\right) .
$$

\subsubsection{Fifth Layer of Energy}

In the fifth layer, the degree of favorable evidence is equal to the degree of unfavorable evidence previously derived. Therefore, $\mu_{(P q L) E 5}=\lambda_{(P q L) E 4}$ or

$$
\mu_{(P q L) E 5}=\sqrt{\sqrt{\sqrt{\frac{H_{(s) P q L}}{2}}}} .
$$

The degree of unfavorable evidence will be obtained through the square root of $\mu_{(P q L) E 5}$. Therefore,

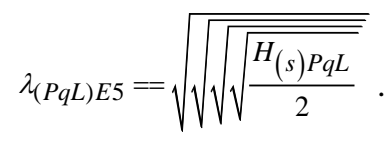

Given the relation to the pure state of the fundamental layer, the degree of unfavorable evidence of the energy level $\left(E_{5}\right)$ of the current state is calculated by multiplication, such that $\lambda_{(P q L) E E 5}=\mu_{(P q L) E 5} \times \lambda_{(P q L) E 5}$ or

$$
\lambda_{(P q L) E E 5}=\sqrt{\sqrt{\sqrt{\frac{H_{(s) P q L}}{2}}}} \times \sqrt{\sqrt{\sqrt{\sqrt{\frac{H_{(s) P q L}}{2}}}}} .
$$

In this case, the degree of certainty for energy level $E_{5}$ can be derived as follows:

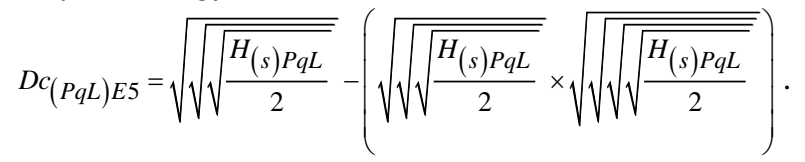

The degree of contradiction for energy level $E_{5}$ can be derived as follows: 


$$
D c t(P q L) E 5=\sqrt{\sqrt{\sqrt{\frac{H_{(s) P q L}}{2}}}}+\left(\sqrt{\sqrt{\sqrt{\frac{H_{(s) P q L}}{2}}}} \times \sqrt{\sqrt{\sqrt{\sqrt{\frac{H_{(s) P q L}}{2}}}}}\right)-1 .
$$

In the fifth layer, the degenerate paraquantum logical state will be represented by the function:

$$
\psi_{(P q L) E 5}=\left(1-D c_{(P q L) E 5}, D c t(P q L) E 5\right)
$$

or

$$
|\psi\rangle=\left(\left(1-D c_{n d(P q L) E 5}\right)\right)|0\rangle+\left(D c t_{n d(P q L) E 5}\right)|1\rangle,
$$

where $D c_{(P q L) E 5}$ is presented in Eq. (58), and $D c_{(P q L) E 5}$ is presented in Eq. (59).

In the fifth layer, the pure or nondegenerate paraquantum logical state will be represented by the functions expressed Eqs. (58) and (27), as follows:

$$
\psi_{(P q L) E 5 \text { pure }}=\left(1-D c_{(P q L) E 5}, D c t(P q L) E 1\right) .
$$

\subsubsection{Sixth Layer of Energy}

In the sixth layer, the degree of favorable evidence is equal to the degree of unfavorable evidence previously derived. Therefore, $\mu_{(P q L) E 6}=\lambda_{(P q L) E 5}$ or

$$
\mu_{(P q L) E 6}=\sqrt{\sqrt{\sqrt{\sqrt{\frac{H_{(s) P q L}}{2}}}}} .
$$

The degree of unfavorable evidence will be obtained through the square root of $\mu_{(P q L) E 6}$. Therefore,

$$
\lambda_{(P q L) E 6}=\sqrt{\sqrt{\sqrt{\sqrt{\sqrt{\frac{H_{(s) P q L}}{2}}}}}} .
$$

The degree of unfavorable evidence of the energy level $\left(E_{6}\right)$ of the current state is calculated by multiplication, such that $\lambda_{(P q L) E E 6}=\mu_{(P q L) E 6} \times \lambda_{(P q L) E 6}$ or

$$
\lambda_{(P q L) E E 6}=\sqrt{\sqrt{\sqrt{\sqrt{\frac{H_{(s) P q L}}{2}}}}} \times \sqrt{\sqrt{\sqrt{\sqrt{\sqrt{\frac{H_{(s) P q L}}{2}}}}} .}
$$

The degree of certainty for energy level $E_{6}$ can be derived as follows:

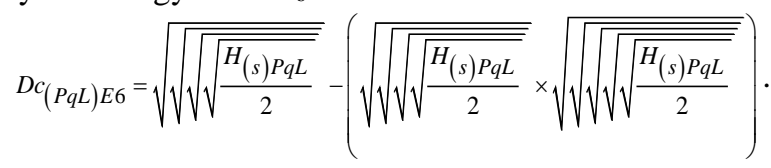

The degree of contradiction for energy level $E_{6}$ can be derived as follows:

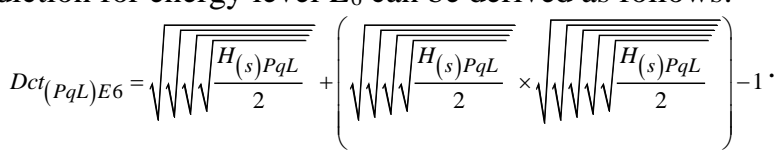

In the sixth layer, the degenerate paraquantum logical state will be represented by the function:

$$
\psi_{(P q L) E 6}=\left(1-D c_{(P q L) E 6}, D c_{(P q L) E 6}\right)
$$

or

$$
|\psi\rangle=\left(\left(1-D c_{(P q L) E 6}\right)\right)|0\rangle+\left(D c t_{(P q L) E 6}\right)|1\rangle,
$$

where $D c_{(P q L) E 6}$ is presented in Eq. (66), and $D c t_{(P q L) E 6}$ is presented in Eq. (67).

In the sixth layer, the pure or nondegenerate paraquantum logical state will be represented by the functions expressed in Eqs. (66) and (27), as follows:

$$
\psi_{(P q L) E 6 \text { pure }}=\left(1-D c_{(P q L) E 6}, D c t(P q L) E 1\right) .
$$


These procedures can be continued for $n$ layers of the paraconsistent model of the atom.

The graphs resulting from the energy layer equations are shown in the results section.

\subsection{PqL Energy Equations for the Observer in the Vector Base $Y$}

For the interlaced $\mathrm{PqL}$ bilattice, the equations that translate this situation can be obtained using the same procedures performed to obtain the equations for calculating the degrees of certainty and contradiction. To derive the equations for the $Y$ observer, we initially consider the same probabilistic function used for the $X$ observer, with its values allocated to the same USCP (lattice $\kappa$ ). To obtain the degrees of certainty and contradiction for the $Y$ observer, we will apply the actions that previously created the transformations that resulted in the degrees of certainty and contradiction, now considering the rotation of $45^{\circ}$ to be clockwise. These actions are as follows: (a) expansion of $\sqrt{2}$ from the $x$ - and $y$-axes $T_{1}\left(X_{1}, Y_{1}\right)=(x \sqrt{2}, y \sqrt{2})$; (b) $45^{\circ}$ clockwise rotation at the origin $T_{2}\left(X_{2}, Y_{2}\right)=\left(X_{1} \cdot \cos \theta+Y_{1} \cdot \sin \theta, X_{1} \cdot \sin \theta-Y_{1} \cos \theta\right)$ where $\cos \theta=\frac{1}{\sqrt{2}}$ and $\sin \theta=\frac{1}{\sqrt{2}} ;$ (c) translation of the -1 value from the $x$-axis $T_{3}\left(X_{3}, Y_{3}\right)=\left(X_{2}-1, Y_{2}\right)$, resulting in $T_{3}\left(X_{3}, Y_{3}\right)=(x+y-1, x-y)$ or

$$
T_{3(p)}\left(X_{3}, Y_{3}\right)=\left(\mu_{(p)}+\lambda_{(p)}-1, \mu_{(p)}-\lambda_{(p)}\right) .
$$

For the $Y$ observer, we denote the contradiction degree $(D c t)$ as $X_{3}$ and the certainty degree $(D c)$ as $Y_{3}$ : $X_{3}=D c \rightarrow$ Certainty degree:

$Y_{3}=D c t \rightarrow$ Contradiction degree:

$$
D c_{(p) y}=\mu_{(p)}+\lambda_{(p)}-1
$$

$$
\operatorname{Dct}_{(p) y}=\mu_{(p)}-\lambda_{(p)} .
$$

Therefore, from Eq. (71) -transformation $T_{3(p)}$, we obtain the certainty degree equation $\left(D c_{(\mu(p), \lambda(p))}\right)$, with

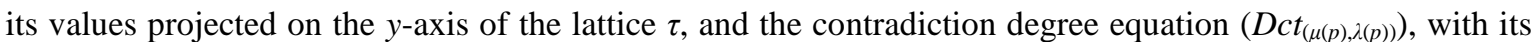
values projected on the $x$-axis of the lattice $\tau$.

In Fig. 4(a), the mapping with the sequences of actions of the paraconsistent transformations for the $Y$ observer is shown.

Given that the paraquantum logical state will have its values changed through the modification of the orbital trajectory, for the $Y$ observer, its representation will be at the extremity of the internal state vector of the $\mathrm{PqL}$, originating from the vertex where the inconsistent logical state is located. For logical negation, the internal state vector will have its origin at the vertex, where the extreme logical state "paracomplete" is located.

Given that the paraquantum logical state $\psi \tau$ is the point of intersection between the degree of certainty $(D c)$ and the degree of contradiction $(D c t)$ located in the interlaced PqL bilattice, the representation of the $Y$ observer in the form of a set of complex numbers will be expressed as follows:

$$
\psi_{\psi \text { int }} I y=i D c_{(p)}+D c t_{(p)} .
$$

An internal state vector with a unitary module and that originates from the inconsistent extreme logical state $(\mathrm{T})$ is derived as follows:

$$
M \psi y=\sqrt{\left(D c_{(p)}\right)^{2}+\left(D c t_{(p)}\right)^{2}} .
$$

This internal state vector with a unitary module defines the orbital paths within the interlaced $\mathrm{PqL}$ bilattice. The same values represent the external logical state for the ground state (level $E_{1}$ ), which can be expressed as follows:

$$
\psi_{\psi \text { int }} I y=i\left(1-D c_{(p) y}\right)+D c t_{(p) y} .
$$

This unitary module of the state vector defines the external orbital trajectory in the PqL bilattice. The external orbital trajectory is made at the extremity of the external state vector with a unitary module and whose origin is at the point equidistant from the vertices of the interlaced PqL bilattice. Its module is expressed as follows:

$$
M \psi I y=\sqrt{\left(1-D c_{(p) y}\right)^{2}+\left(D c t_{(p) y}\right)^{2}} .
$$


The values representing the external logical state of the ground state (level $E_{1}$ ) for the $Y$ observer with the probability values used in the $\mathrm{PqL}$ can be derived as follows:

$$
\psi_{\psi y}=\left(D c_{(p) y}, D c t_{(p) y}\right) .
$$

Figure 4(b) shows Quadrant I of the interlaced PqL bilattice with the state vectors and orbit trajectories of the $Y$ observer.

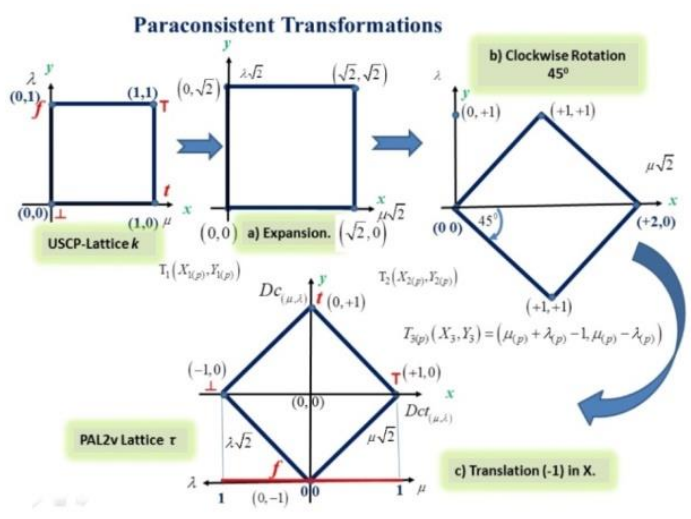

(a)

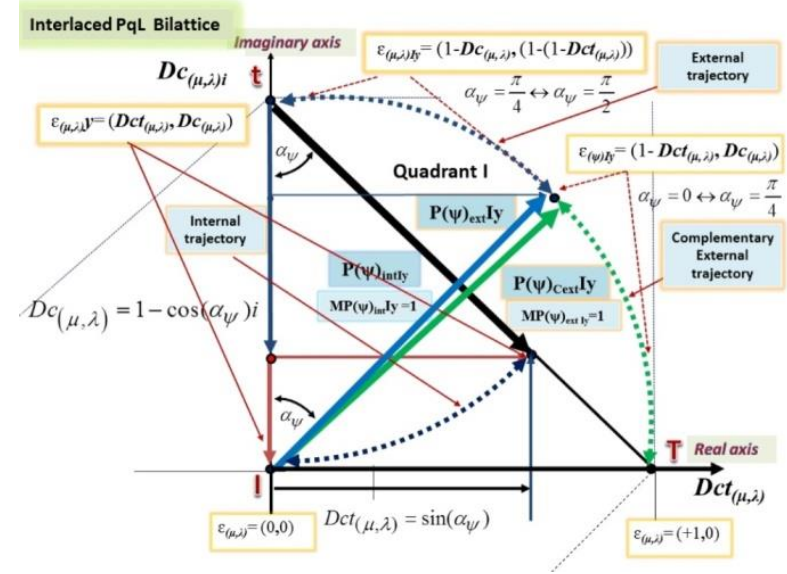

(b)

Fig. 4. Paraconsistent transformations and state vectors for the $Y$ observer: (a) sequences of the paraconsistent transformations for the $Y$ observer with clockwise rotation; (b) Quadrant I of the interlaced PqL bilattice with the vectors and orbit trajectories of the $Y$ observer.

\subsection{A Representation of the Paraconsistent Model of the Atom}

The mapping sequences of probabilistic evidence degrees with both the $X$ and $Y$ observers result in equations of superposed paraquantum logical states in Quadrant I of the interlaced PqL bilattice. Using the paraquantum equations, we can present the results of the superposed logical states as two bilattices comprising one superposed plane.

\subsubsection{Superposed Paraconsistent Logical States}

The energy equations for the $Y$ observer are represented by the quantum logical states with a set of complex numbers, where the imaginary and real values will change depending on the observer. For the $Y$ observer, the vector base will be orthogonal to the base $X$. This means that, for the $Y$ observer, the imaginary values of the $X$ observer will be their real values and the actual values of the $X$ observer will be their imaginary values.

Figure 5(a) shows the sequences of the paraconsistent transformations for the $X$ and $Y$ observers and framework vectors.

Figure 5(b) shows the interlaced PqL bilattice and the vectors and trajectories of the $X$ and $Y$ observers with superposed paraquantum logical states.

In the next section, a paraconsistent model of the atom is constructed with PqL equations formalized with the Shannon entropy function. With the range of probability values (p), we will obtain the degrees of evidence and the degrees of certainty and contradiction forming the paraquantum logical states and energy values to show through the graphical results the individual behavior and its representations that simulate quantum phenomena. 


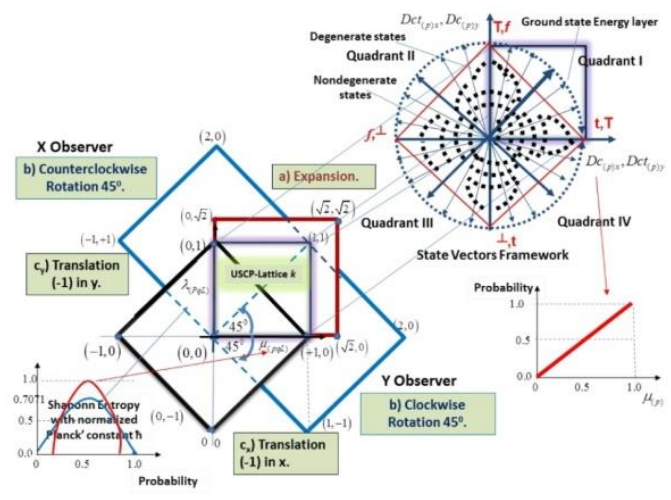

(a)

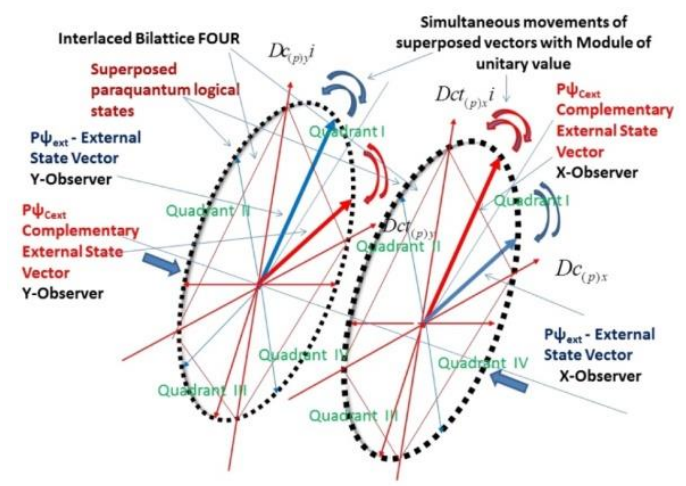

(b)

Fig. 5. Superposed paraconsistent logical states and probabilistic trajectories of the $X$ and $Y$ observers: (a) sequences of the paraconsistent transformations for the $X$ and $Y$ observers and framework vectors; (b) interlaced bilattices with the vectors and orbit trajectories of the $X$ and $Y$ observers with superposed paraquantum logical states.

In the paraconsistent model, the equations form the superposed paraquantum logical states located in the planes of the $X$ and $Y$ observers. Moreover, variations of the probability values are applied to the equations expressing the orbital trajectories of the particles in the two superposed planes as traces of energy in the overlapping layers of the atom.

\subsection{Algorithms and Testing Procedures}

For the simulation of the paraconsistent model of the atom we consider that the functions that generate degrees of evidence, certainty and contradiction are discrete. Therefore, it is necessary to specify a number of $\mathrm{N}$ iterations for the algorithm. If we consider that this number $\mathrm{N}$ is related to a unit of time, then it means the frequency of the appearance of the paraquantum logical states located at the end of the state vector with a unitary module $(\mathrm{P} \psi)$. The inverse of this frequency is the period $\mathrm{T}$, therefore the interval $(\Delta p)$ of each iteration that produces the movement of the state vector in the interlaced PqL bilattice. The minimum number of iterations has been specified in 10 and the maximum number of iterations be only limited by the computational capacity. In the algorithm, the iterations count in ascending order, starts at 1 and ends at Nmax-1. In descending order, it starts at Nmax-1 and ends at Nmax-(Nmax- $\Delta p)$. Thus, the probability value $p$ in the Shannon function will never be 0 or 1 . As the number of iterations $\mathrm{N}$ specified for the generation of degrees of evidence can be a minimum value of 10 and an infinite maximum value, then in the paraconsistent model of the atom there can be an infinite number of state vectors with unitary module, where, in relation to frequency, all state vectors are different from each other.

In this work, we will present two main algorithms that are two generator systems of degrees of evidence, certainty and contradiction. Both algorithms are used in the simulations to obtain the graphical results regarding the study of the behavior of the degrees of evidence and the trajectories of the paraquantum logical states in a representation of the atom in the ground-state with the observer in $\mathrm{X}$.

In the simulations with fundamental PqL-equations and for energy layers of the hydrogen atom, the number of sequential steps used was $\operatorname{Nmax}=100$, therefore the probability values $(p)$ are with intervals $\Delta p$ in the order of $1 / 100$. The value used as the Shannon normalization factor was $1=1.057402554$, and the pi constant used was $\pi=3.141592654$. 


\subsection{Generating degrees of evidence with the Shannon entropy function}

In the construction of the model, probability $p$ is defined as the system input value and the PqLevidence

degrees are obtained through the Shannon entropy function. In algorithm application the probability is originated from two sources of information that generate data simultaneously.

Source 1 . For probability p ranging between 0 and 1 .

Source 2. For probability p ranging between 1 and 0 .

\section{PqL-ALGORITHM 1 - Generator of degrees of evidence with Shannon function application}

Function PqLEvidShanEntropy (in: $p_{(n)_{+}}, q_{(n)_{+}}, p_{(n)_{-}}, q_{(n)_{-}}$out: $\left.\mu_{(P q L) i n c r}, \lambda_{(P q L) i n c r}, \mu_{(P q L) \text { decr }}, \lambda_{(P q L) d e c r}\right)$

1. Give the number of sequential steps used $\left(10 \leq N_{\max } \leq\right.$ Computational limit $): N_{\max }=\ldots \ldots$

1.1 Calculate the probability variation value $(\Delta p)$ used in each iteration: $\Delta p=\frac{1}{N_{\max }}$

2. Consider the iteration count starting at 1 and ending at (Nmax-1) and calculate the sequence values of the probability in increasing order $p_{(n)_{+}}$:

$$
p_{(n i)_{+}}=\left\{1 \Delta p, 2 \Delta p, 3 \Delta p, \ldots,\left(N_{\max } \Delta p\right)-\Delta p\right\}
$$

2.1 Calculate the complementary values $q_{(n)_{+}}: \quad q_{(n i)_{+}}=1-p_{(n i)_{+}}$

2.2 Calculate the Shannon Entropy function values in increasing order (Eq.(23)):

$$
H_{(s) P q L_{i n c r}}=-1 \pi\left[p_{(n i)_{+}} \log p_{(n i)_{+}}+q_{(n i)_{+}} \log q_{(n i)_{+}}\right]
$$

3. Consider the iteration count starting at (Nmax-1) and ending at Nmax-(Nmax- $\Delta p)$ and calculate the sequence values of the probability in decreasing order $p_{(n i)_{-}}$:

$$
p_{(n i)_{-}}=\left\{\left(N_{\max } \Delta p\right)-1 \Delta p,\left(N_{\max } \Delta p\right)-2 \Delta p,\left(N_{\max } \Delta p\right)-3 \Delta p, \ldots,\left(N_{\max } \Delta p\right)-\left(N_{\max }-\Delta p\right) \Delta p\right\}
$$

3.1 Calculate the complementary values complementary values $q_{(n i)_{-}}: q_{(n i)_{-}}=1-p_{(n i)_{-}}$

3.2 Calculate the Shannon Entropy function values in decreasing order $H_{(s) P q L i n c r}$ (Eq.(23)):

$$
H_{(s) P q L_{\text {decr }}}=-1 \pi\left[p_{(n i)_{-}} \log p_{(n i)_{-}}+q_{(n i)_{-}} \log q_{(n i)_{-}}\right]
$$

4. Present the favorable evidence degree of increasing order (Eq. (24)): $\mu_{(P q L) i n c r}=H_{(s) P q L_{\text {incr }}}$

4.1 Calculate the unfavorable evidence degree of increasing order (Eq. (25)):

$$
\lambda_{(P q L) i n c r}=\sqrt{\frac{H_{(s) P q L_{\text {incr }}}}{2}}
$$

5. Present the favorable evidence degree of decreasing order (Eq. (24)): $\mu_{(P q L) \text { decr }}=H_{(s) P q L_{\text {decr }}}$

5.1 Calculate the unfavorable evidence degree of decreasing order (Eq.(25)):

$$
\lambda_{(P q L) d e c r}=\sqrt{\frac{H_{(s) P q L_{d e c r}}}{2}}
$$

6. Plot on the same chart: $p \times \mu_{(P q L) i n c r}, p \times \lambda_{(P q L) i n c r}, p \times \mu_{(P q L) d e c r}$ and $p \times \lambda_{(P q L) d e c r}$.

7. Return to step 1.

In the paraconsistent model of the atom, all equations are constructed with degrees of evidence, so, from this main algorithm, it is possible to plot other results by applying the equations presented in the text.

2.12 PqL Algorithm for generating paraquantum logical states with the Shannon entropy function 


\section{PqL-ALGORITHM 2 - Paraquantum logical States Generator with Shannon Function application}

Function PqLstatesShanEntropy (in: $H_{(s) P q L_{i n c r}}, H_{(s) P q L_{\text {decr }}}$ out: $\left.\psi_{(P q L)} P q L s t a t e s\right)$

1. For each value of the Shannon functions $H_{(s) P q L_{\text {incr }}}$ and $H_{(s) P q L_{\text {dec }}}$ :

1.2 Calculate the probabilistic certainty degree of increasing order in the ground state (Eq. (26)):

$$
D c_{(P q L) \text { incr }}=H_{(s) P q L_{\text {incr }}}-\sqrt{\frac{H_{(s) P q L_{\text {incr }}}}{2}}
$$

1.3 Calculate the probabilistic contradiction degree of increasing order in the ground state (Eq. (27)):

$$
\operatorname{Dct}_{(P q L) i n c r}=H_{(s) P q L_{\text {incr }}}+\sqrt{\frac{H_{(s) P q L_{\text {incr }}}}{2}}-1
$$

1.4 Calculate the probabilistic certainty degree of decreasing order in the ground state (Eq. (26)):

$$
D c_{(P q L) d e c r}=H_{(s) P q L_{d e c r}}-\sqrt{\frac{H_{(s) P q L_{\text {decr }}}}{2}}
$$

1.5 Calculate the probabilistic contradiction degree of decreasing order in the ground state (Eq. (27)):

$$
\operatorname{Dct}_{(}(P q) \operatorname{decr}=H_{(s) P q L_{\text {decr }}}+\sqrt{\frac{H_{(s) P q L_{\text {decr }}}}{2}}-1
$$

2. Present the Paraquantum logic state functions for quadrant I (Eq. (28)):

Increasing order: $\psi_{(P q L) i n c r} I=\left(1-D c_{(P q L) i n c r}, D c t(P q L) i n c r^{i}\right)$

Decreasing order: $\psi_{(P q L) d e c r} I=\left(1-D c_{(P q L) \operatorname{dec} r}, D c t(P q L) d e c r i\right)$

2.1 Present the complementary values (Eq. (17)):

$$
\begin{aligned}
& \text { Increasing order: } \uparrow_{\psi_{(P q L) i n c r} I}=\left(1-D c t_{(P q L) i n c r}, 1-\left(1-D c_{(P q L) i n c r}\right) i\right) \\
& \text { Decreasing order: } \left.\uparrow_{\psi_{(P q L) d e c r} I=\left(1-D c t_{(}(P L)\right) \operatorname{dec}, 1-\left(1-D c_{(P q L) d e c r}\right) i}\right)
\end{aligned}
$$

3. Apply the Negation Operator for obtain values to quadrant II (Eq. 9):

Increasing order: $\psi_{(P q L) i n c r} I I=\left(D c_{(P q L) i n c r}-1, D c t(P q L) i n c r i\right)$

$$
\text { Decreasing order: } \psi_{(P q L) d e c r} I I=\left(D c_{(P q L) \operatorname{decr}}-1, D c t(P q L) \text { decr } i\right)
$$

3.1 Present the complementary values:

$$
\begin{aligned}
& \text { Increasing order: } \psi_{(P q L) i n c r} I I=\left(D c t_{(P q L)} \text { incr }-1,-D c_{(P q L) i n c r}{ }^{i}\right) \\
& \text { Decreasing order: } \psi_{(P q L) \text { decr }} I I=\left(D c t_{(P q L) \text { decr }}-1,-D c_{(P q L) \text { decr }} i\right)
\end{aligned}
$$

4. Apply the Complementation Operator for obtain values to quadrant III (Eq. 10):

Increasing order: $\psi_{(P q L) i n c r} I I I=\left(D c_{(P q L) i n c r}-1,-D c t(P q L) i n c r i\right)$

$$
\text { Decreasing order: } \psi_{(P q L) d e c r} I I I=\left(D c_{(P q L) d e c r}-1,-D c t(P q L) d e c{ }^{i}\right)
$$

4.1 Present the complementary values:

$$
\begin{aligned}
& \text { Increasing order: } \psi_{(P q L) i n c r} I I I=\left(D c t_{(P q L) i n c r}-1,1-\left(1-D c_{(P q L) i n c r}\right) i\right) \\
& \text { Decreasing order: } \psi_{(P q L) d e c r} I I I=\left(D c t(P q L) \text { decr }-1,1-\left(1-D c_{(P q L) d e c r}\right) i\right)
\end{aligned}
$$

5. Apply the Conflation Operator for obtain values to quadrant IV (Eq. 11):

$$
\begin{aligned}
& \text { Increasing order: } \psi_{(P q L) i n c r} I V=\left(1-D c_{(P q L) i n c r},-D c t(P q L) \text { incr }{ }^{i}\right) \\
& \text { Decreasing order: } \psi_{(P q L) d e c r} I V=\left(1-D c_{(P q L) d e c r},-D c t(P q L) \text { decr } i\right)
\end{aligned}
$$

5.1 Present the complementary values:

Increasing order: $\psi_{(P q L) i n c r} I V=\left(1-D c t(P q L) i n c r, D c_{(P q L) i n c r} i\right)$

Decreasing order: $\psi_{(P q L) d e c r} V=\left(1-D c t(P q L) \operatorname{dec}, D c_{(P q L) d e c r} i\right)$ 
6. Consider the representation in complex number set $\psi_{(P q L)}=(\operatorname{Re}, \mathrm{Im})$ and plot on the same chart the results of the all 16 paraquantum logical state functions.

7. Return to step 1.

\subsection{Rydberg energy and Hydrogen atom}

In this work, the paraconsistent model of the atom constructed with the probabilistic function of Shannon's entropy will be correlated to the hydrogen atom through the Rydberg formula. In this way, the energy values obtained in the paraconsistent atom model simulation are all correlated with the values defined in Eq. (13); therefore, for application in the hydrogen atom, the Rydberg energy is the unit for energy and is calculated by $E_{R y d}=\frac{m_{e} e^{4}}{2\left(4 \pi \epsilon_{0}\right)^{2} \mathrm{~h}^{2}}=R_{\infty} h c$. Here, the Rydberg constant is $R_{\infty}=10973731.568527 m^{-1}, h$ is the Planck's constant, and $c$ is the speed of light. For this condition the Rydberg energy is $E_{R y d}=R_{\infty} h c=2.179872085 \times 10^{-18} \mathrm{~J}$. With $e=1.60217653 \times 10^{-19} \mathrm{C}$, then $E_{R y d}=13.60569225 \mathrm{eV}$. In the simulations, this value will be multiplied by the degrees of certainty equations to obtain the energy values in each layer of the hydrogen atom.

\section{Results}

The graphical results that will be presented are from simulations using the PqL equations in a calculi spreadsheet.

\subsection{Results Related to the Fundamental PqL-Equations of the Paraconsistent Model of the Atom}

With application of PqL-ALGORITHM 1: Figure 6(a) shows the graphs of the results of the degree of favorable evidence $\mu_{(\mathrm{PqL})}$ derived using Eq. (24) and the degree of unfavorable evidence $\lambda_{(\mathrm{PqL})}$ derived using Eq. (25). With application of PqL-ALGORITHM 2: Figure 6(b) shows the variations of the degrees of certainty (Eq. 26) and contradiction (Eq. 27) and the simulation results of the unitary module $\mathrm{M}(\psi) \mathrm{I}_{1}$ of the quantized probabilistic function (Eq. 29) for a complete variation of probability $p$.

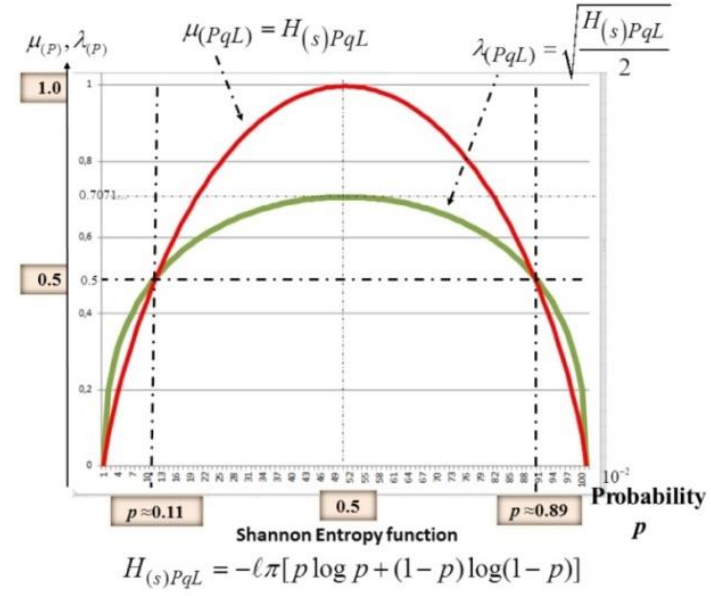

(a)

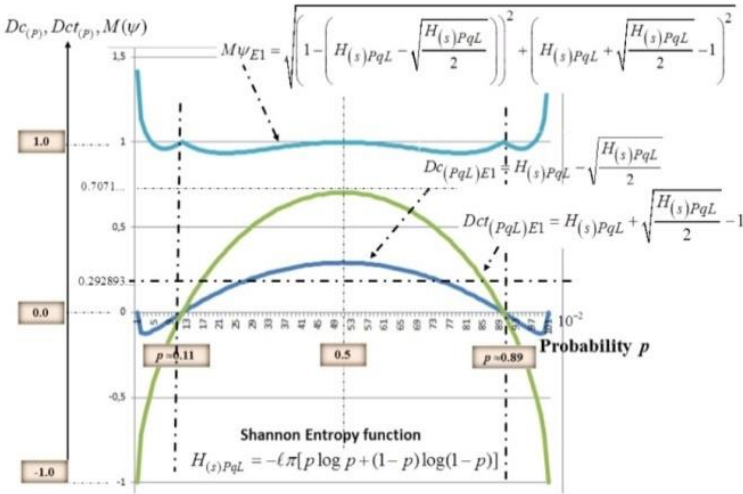

(b)

Fig. 6. Results related to the fundamental PqL-equations: (a) graphs of the results of the degrees of favorable evidence $\mu_{\text {(p) }}$ (Eq. 24) and unfavorable evidence $\lambda_{\text {(p) }}$ (Eq. 25); (b) simulation results of the degrees of certainty and contradiction, according to Eqs. (26) and (27), respectively, and the unitary module $\mathrm{M}(\psi) \mathrm{I}_{1}$ of the quantized probabilistic function (Eq. 29) for a complete variation of probability $p$. 


\subsection{Results Related to the Ground-State Energy of the Paraconsistent Model of an Atom}

With application of PqL-ALGORITHM 2: Figure 7(a) shows the simulation results of the ground-state energy for the paraconsistent model of the atom.

With application of PqL-ALGORITHM 2: Figure 7(b) shows the simulation results with explications about the utilized equations, and the interlaced $\mathrm{PqL}$ bilattice circumscribed in the external orbital circumference. In this simulation, we used Eqs. (24) to (27) and the logical operations of negation, complementation, and conflation.

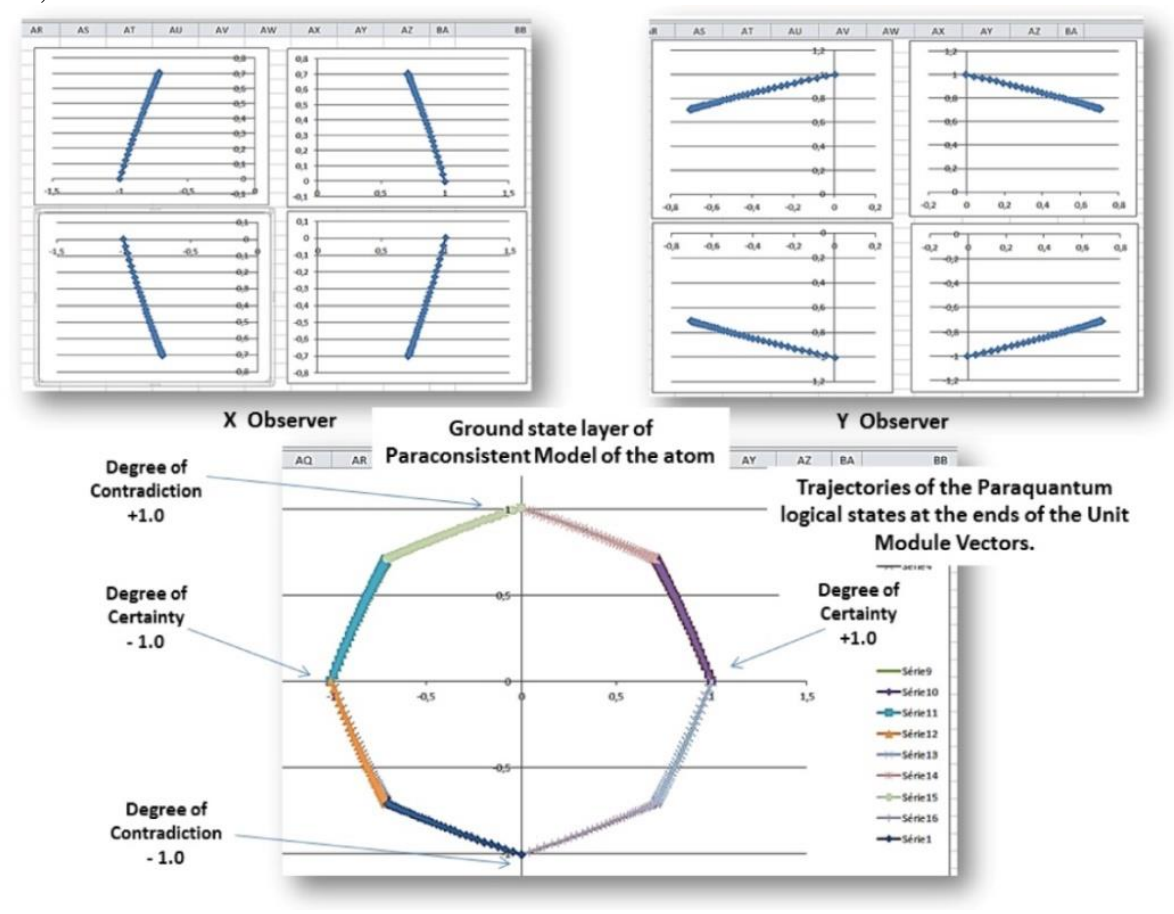

(a)

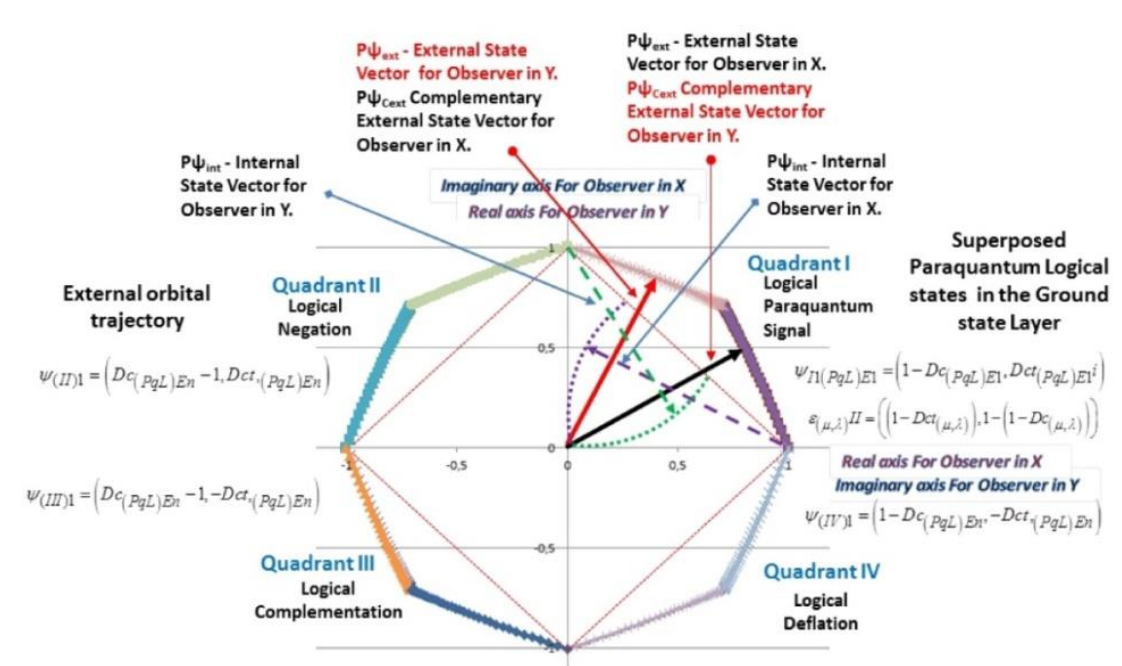

(b)

Fig. 7. Results related to the fundamental PqL-equations for the energy layer. (a) Simulation of the groundstate energy of the atom using the equations of Quadrant I and the PqL operators. (b) Simulation results with explications about the utilized equations and the interlaced $\mathrm{PqL}$ bilattice circumscribed in the external orbital circumference. 
Figure 8(a) shows the results simulated with a group of Shannon entropy functions, which are obtained using the equations of the degrees of certainty in six layers of the paraconsistent model of the atom for nondegenerate states. The results of the simulations obtained using the equations defining the six layers of the paraconsistent model of the atom for the degenerate state mode from the $X$ observer are shown in Fig. 8(b).

Figure 8(c) shows the representation of the $\mathrm{PqL}$ bilattice, including the results for the degenerate state mode in the paraconsistent model of the atom from the $X$ observer. In this simulation, we used Eqs. (24) to (27), (33) to (35), (39) to (43), (47) to (51), (55) to (59), and (63) to (67) and the logical operations of negation, complementation, and conflation.

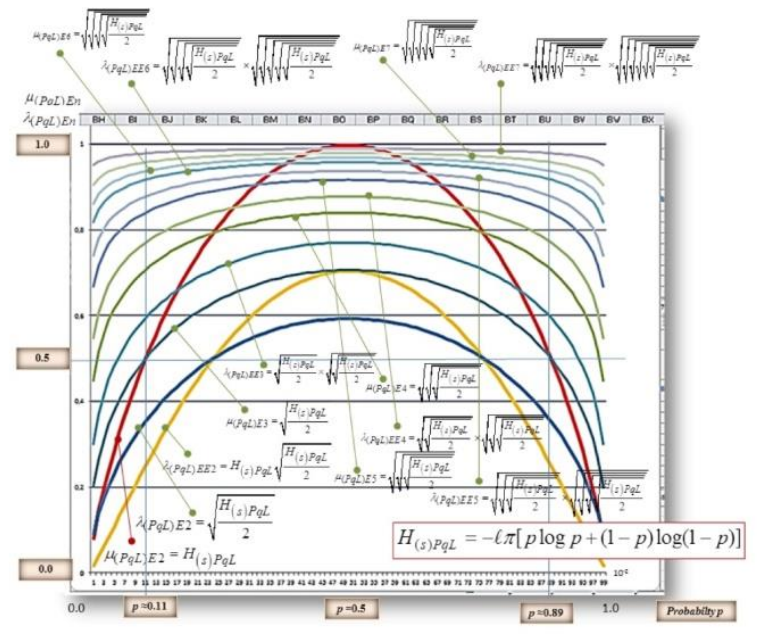

(a)

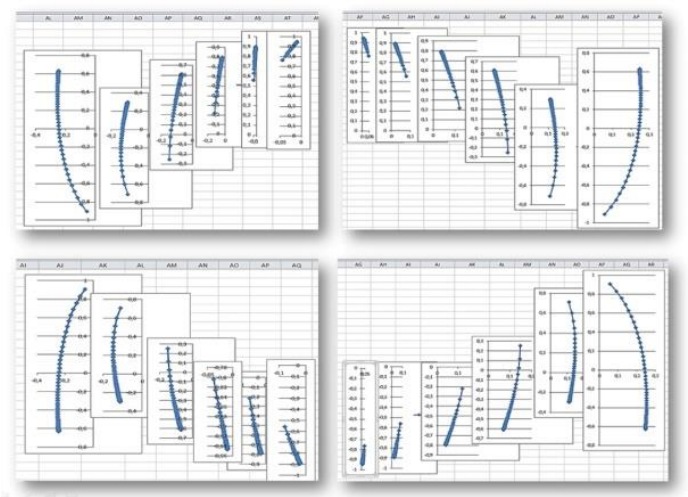

(b)

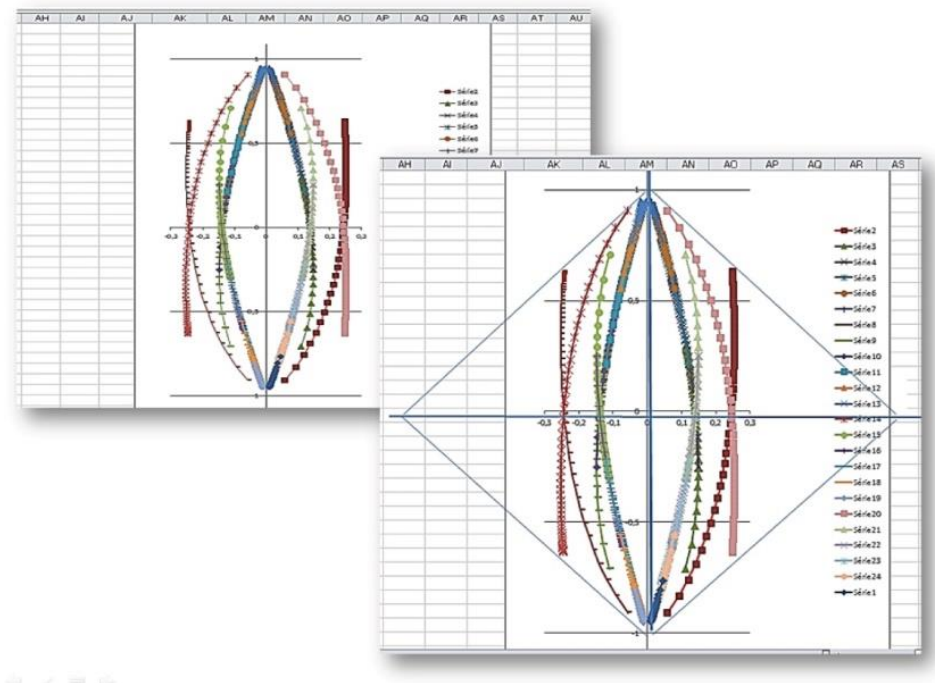

(c)

Fig. 8. Graphs resulting from the energy layer with fundamental PqL-equations from the $X$ observer: (a) simulation results obtained using a group of Shannon entropy functions for calculating the certainty degree equations in six layers of the paraconsistent model of the atom for the pure or nondegenerate states; (b) simulation of the energy layer with degenerate states of the atom using the paraquantum equations in the mode from the $X$ observer; (c) representation of the PqL bilattice, including the results for the degenerate state mode in the paraconsistent model of the atom from the $X$ observer. 
The simulation results obtained using the PqL equations defining the six layers of the paraconsistent model of the atom for the degenerate state mode from the $Y$ observer are shown in Fig. 9(a).

Figure 9(b) shows the representation of the simulation of the degenerate states of the atom in the mode from the $Y$ observer, with the inclusion of the PqL bilattice. In this simulation, we used the adapted equations shown in the "PqL Energy Equations for the $Y$ Observer" section, with the logical operations of negation, complementation, and conflation.

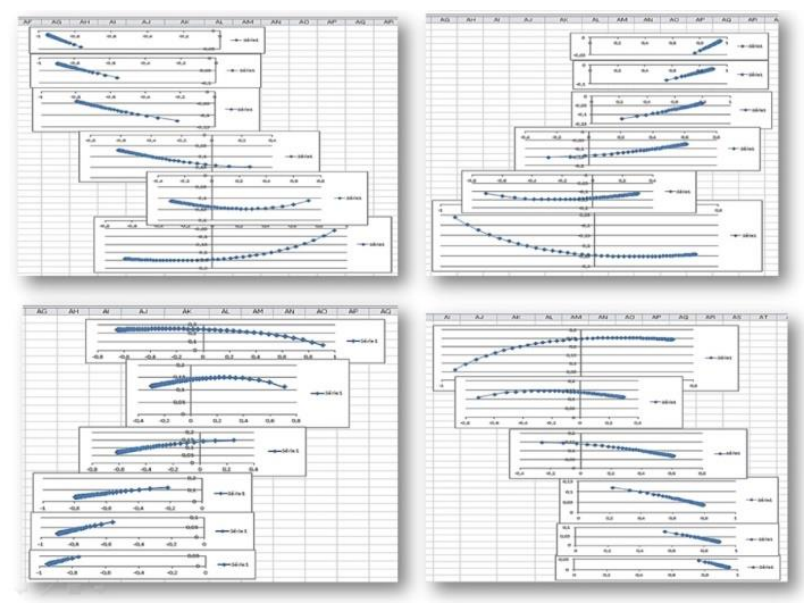

(a)

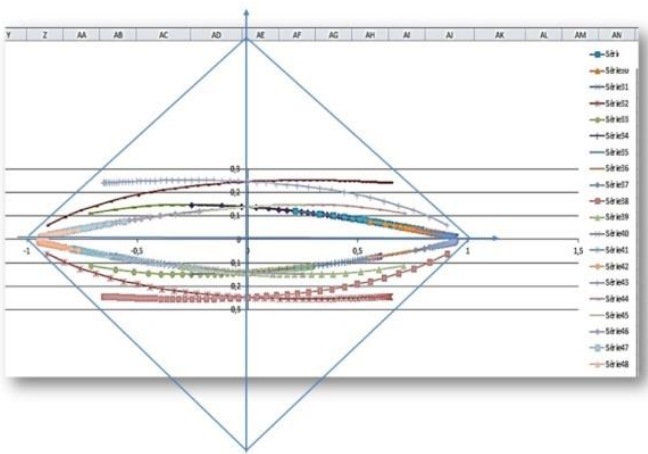

(b)

Fig. 9. PqL Energy fundamental PqL-equations for the $Y$ observer: (a) simulation of the energy layers with degenerate and nondegenerate states of the atom using the paraquantum equations in the mode from the $Y$ observer; (b) representation of the simulation of the degenerate states of the atom in the mode from the $Y$ observer, with the inclusion of the PqL bilattice.

Figure 10(a) shows the results of the simulation of a complete paraconsistent model of the atom without nondegenerate states.

Figure 10(b) shows the simulation results with explications about the utilized equations and the interlaced $\mathrm{PqL}$ bilattice circumscribed in the orbital circumference and energy levels.

Figure 10(c) shows the paraconsistent model of the complete atom with the orbital energy paths of the layers represented by the degrees of certainty and contradiction for the two references of the $X$ and $Y$ observers. In this simulation, we used Eqs. (24) to (76) and the adapted equations shown in the "PqL Equations for the $Y$ Observer" section, with the logical operations of negation, complementation, and conflation. 


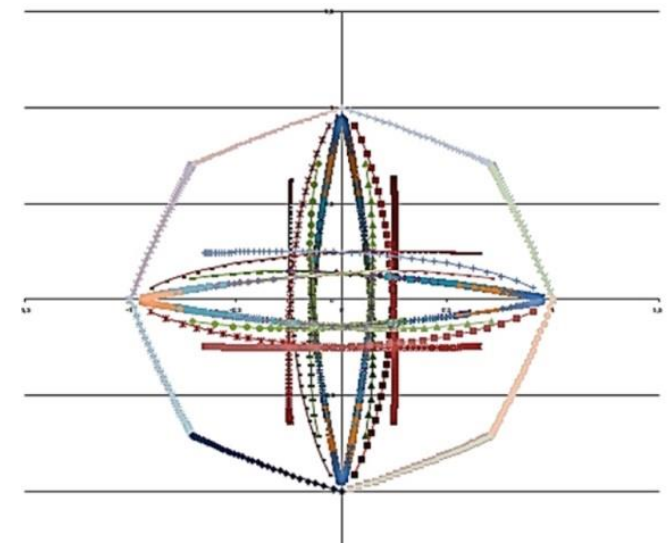

(a)

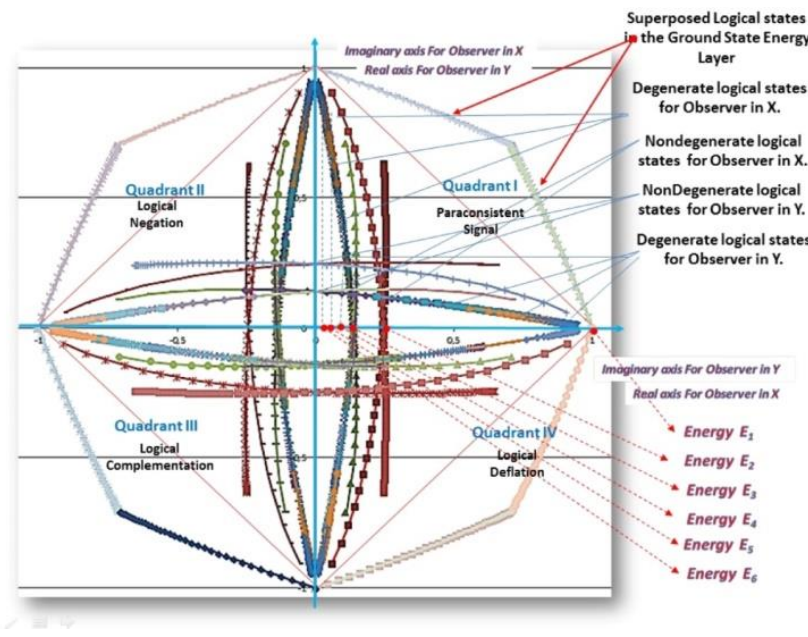

(b)

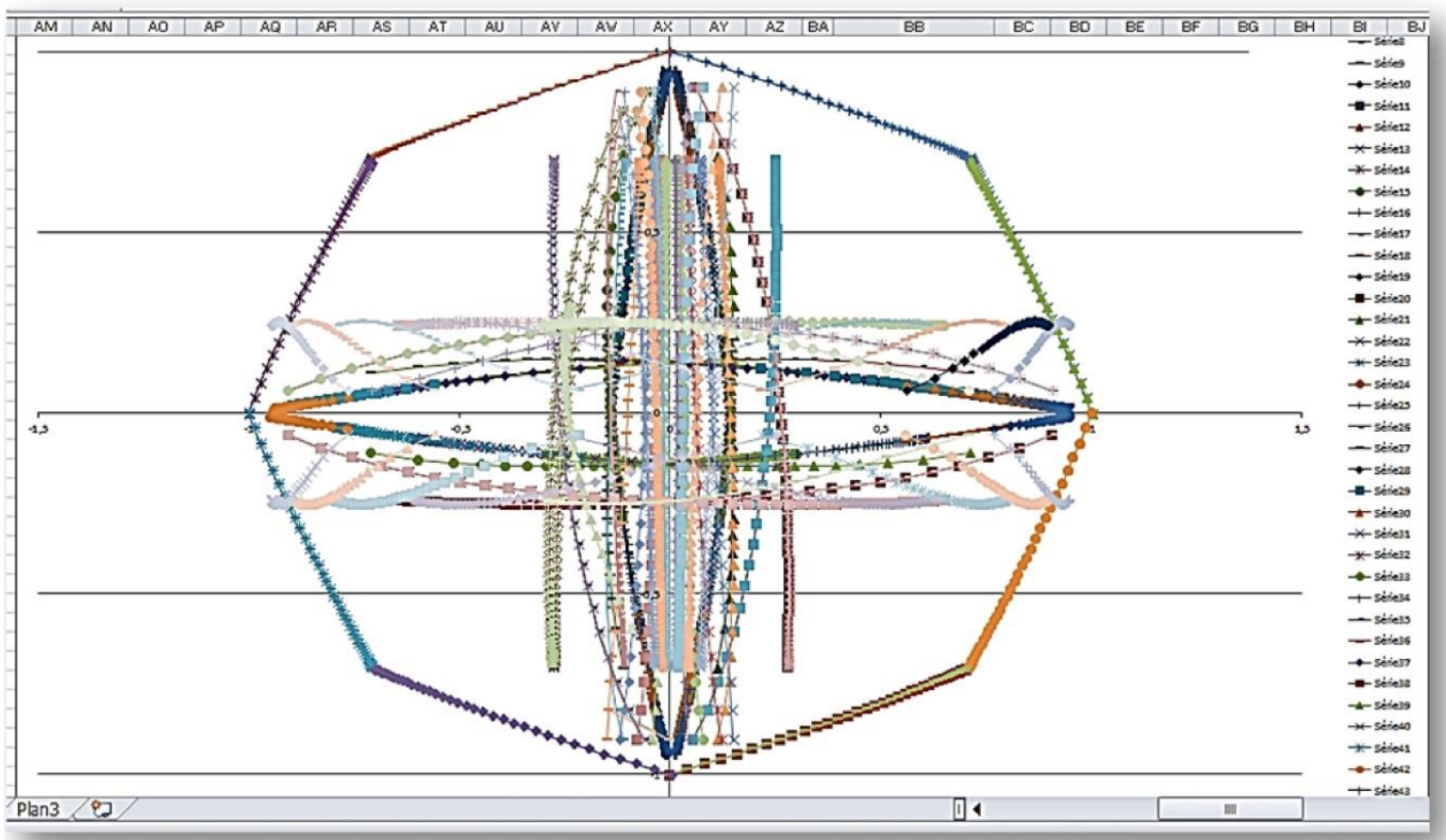

(c)

Fig. 10. The complete paraconsistent model of the atom: (a) simulation results of the paraconsistent model of the atom without nondegenerate states, with the representation of the interlaced PqL bilattice; (b) simulation results of the complete paraconsistent model of the atom with degenerate and nondegenerate states; (c) simulation results of the complete paraconsistent model of the atom with all probabilistic trajectories of the particles in the six equated layers.

3.3 Results Related to the Wave Functions in the Paraconsistent Model of the Atom

With the probabilistic variables established by the PqL equations in which Shannon entropy functions are included, the $D c_{(P q L)}$ and $D c_{(P q L)}$ values are transformed into functions representing the probability emanating from the logical model of the atom. The wave function for each layer of the paraconsistent model of the atom is derived by multiplying the paraquantum logical state with the conjugate complex, according to 
Eq. (2). For the ground-state energy, the wave function $|\psi|^{2}$ is calculated using $\left|\psi_{\left(\mu_{(P q L)}, \lambda_{(P q L)}\right)}\right|^{2}=\psi_{(P q L) E 1} \times \psi^{*}{ }_{(P q L) E 1}$ or

$$
\left|\psi_{\left(\mu_{(P q L)}, \lambda_{(P q L)}\right)}\right|^{2}=\left[\left(1-D c_{(P q L) E 1}\right)+D c t(P q L) E 1^{i}\right] \times\left[\left(1-D c_{(P q L) E 1}\right)-D c t_{(P q L) E 1^{i}}\right]
$$

where $D c_{(P q L) E 1}$ is presented in Eq. (26), and $D c_{(P q L) E 1}$ is presented in Eq. (27).

For the other atomic shells, the axes of the references and the amplitudes of the degrees of certainty and contradiction derived by the equations will be considered.

The graphs obtained by the applications of the equations that are related to the wave functions are shown in Fig. 11(a). Figure 11(b) shows the six wave functions represented in the paraconsistent model of the atom with orbital energy paths of layers $E_{1}, E_{2}$, and $E_{3}$ for the $X$ and $Y$ observers.

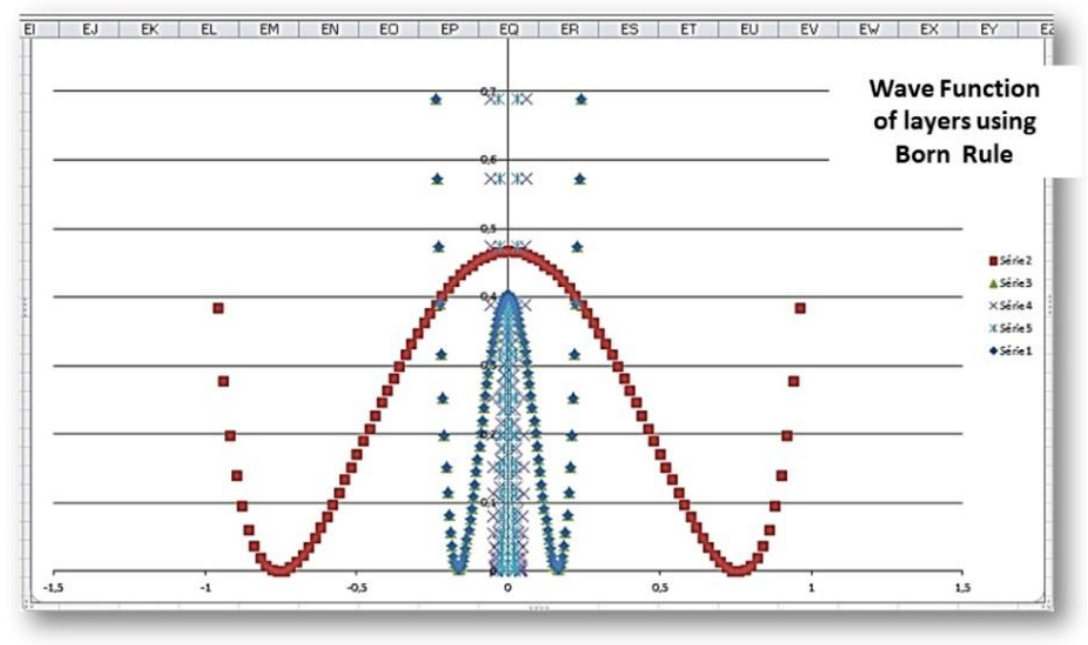

(a)

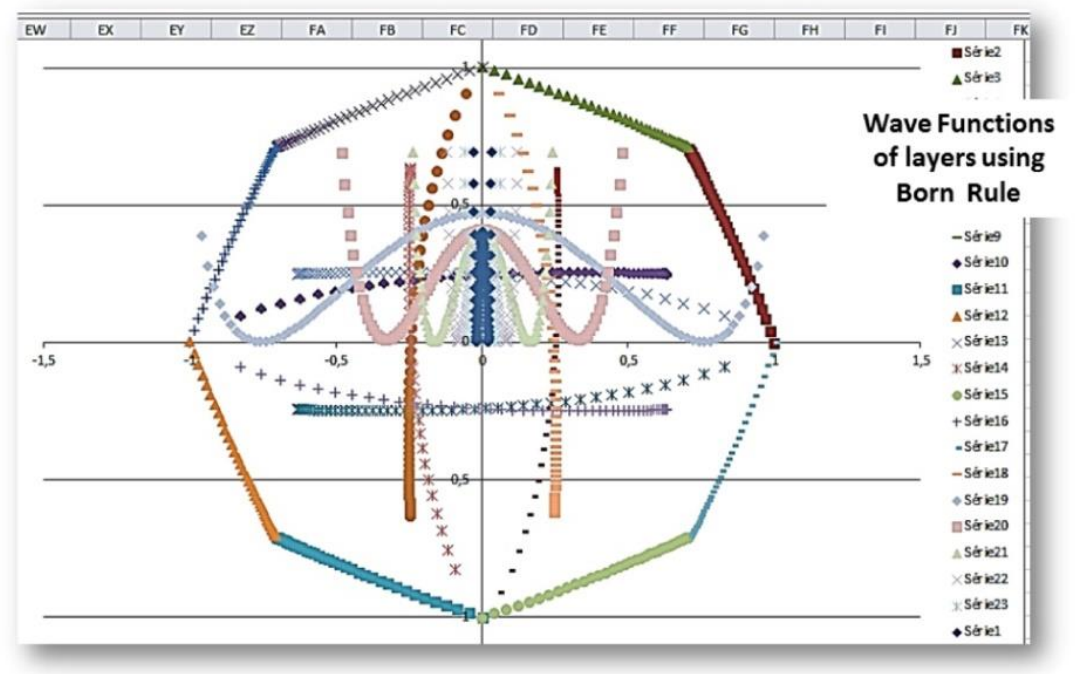

(b)

Fig. 11. Wave functions: (a) simulation results of the wave functions for the ground-state energy and the wave functions for some energy layers; (b) wave functions represented in the paraconsistent model of the atom with orbital energy paths of layers $E_{1}, E_{2}$, and $E_{3}$ for the $X$ and $Y$ observers. 


\subsection{Results Related to the Heisenberg Uncertainty Principle in the PqL}

The reversibility characteristic of the PqL ensures that the degrees of evidence of probability can be obtained through Eqs. (7) and (8). The certainty degrees at the $x$-axis $\left(D c_{(P q L) E}\right)$ and the contradiction degrees at the $y$-axis $\left(D_{\left(t_{(} P L\right) E}\right)$ in the associated interlaced PqL bilattice can be mapped back to the USCP, where the degrees of evidence $\mu_{(\mathrm{p})}$ and $\lambda_{(\mathrm{p})}$ are plotted in the $x$ - and $y$-axes. For the typical paraquantum logical state, that is, $\psi_{(P q L)}=\left(D c_{(P q L)}, D c t_{(P q L)}\right)$, the degree of favorable evidence when the value of the degree of contradiction is 0 can be calculated using $\mu_{(p)}=\frac{1}{2} D c_{(\mu, \lambda)}+\frac{1}{2}$, which is the normalized value for $D c_{(P q L) E}$. With this procedure, the normalized value for the contradiction degree is obtained using $\mu_{(p)}=\frac{1}{2} D c t_{(\mu, \lambda)}+\frac{1}{2}$. We can make this normalization for the external paraquantum logical state of the type $\psi_{(P q L)}=\left(1-D c_{(P q L)}, D c t_{(P q L)}\right)$, where the normalized value for $1-D c_{(P q L)}$ is considered the uncertainty for the $x$-axis, computed as:

$$
\Delta x_{(P q L)}=\frac{\left(1-D c_{(P q L)}\right)+1}{2},
$$

and the normalized value for $D_{t}(P q L)$ is considered the uncertainty for the $y$-axis, computed as:

$$
\Delta y_{(P q L)}=\frac{D c t(P q L)+1}{2} .
$$

With Eqs. (80) and (81), the formal inequality of the Heisenberg uncertainty principle expressed in Eq. (1) is represented in the $\mathrm{PqL}$ as follows:

$$
\Delta x_{(P q L)} \Delta y_{(P q L)} \geq \frac{\mathrm{h}}{2} .
$$

The graphical results of the simulations with the Heisenberg uncertainty principle equations are shown in Fig. 12(a).

\subsection{Results Related to the Calculation of the Probability Value in the Paraconsistent Model of the Atom}

The PqL is a reversible logic, and in this manner, we can analyze the paraconsistent model of the atom from its inner part, considering the nucleus as the energy generator that spreads its values to its external part. With the equations considered in this manner, we can estimate the probable values of energy, as well as the probable location of the energy around the generating nucleus. The reversibility characteristic of the PqL ensures that the degrees of evidence of probability can be obtained through Eqs. (7) and (8), and the result of its normalized value can be compared with the Bernoulli's probabilistic function presented in Eq. (14). This comparison shows that the following equality is a good approximation: $\mu_{(p)}=2 \sigma$, where $\sigma$ is the standard deviation of probabilistic measures. With $\sigma=\frac{\mu_{(p)}}{2}$ and variance $\operatorname{Var}=\sigma^{2}$, the variance in the PqL can be defined as $\operatorname{Var}=\left(\frac{\mu_{(p)}}{2}\right)^{2}=\frac{\mu_{(p)}}{4}$. The variance related to the degree of unfavorable evidence can be computed using $\operatorname{Var}=\lambda_{(p)}{ }^{4}$. In this case, the variance can be represented by the values of the degrees of certainty and contradiction, such that

$$
\operatorname{Var}_{(P q L)}=\left(\frac{-D c_{(P q L)}+D c t(P q L)}{2}\right)^{4} .
$$

From Eq. (12), we can obtain the $p$ values by combining the variance equation with a second-degree equation and applying Bhaskara's method, such that 


$$
p=\frac{1+\sqrt{1-\left[4 \operatorname{Var}_{(P q L)}\right]}}{2} .
$$

For the complement, $q=1-p$ or

$$
q=\frac{1-\sqrt{1-\left[4 \operatorname{Var}_{(P q L)}\right]}}{2} .
$$

Figure 12(a) shows the results of the simulation of the Heisenberg uncertainty principle based on Eqs. (80), (81), and (82). Figure 12(b) shows the results of the simulation of Eqs. (84) and (85), with probability values ranging from 0 to 1.0 as $p$ and from 1.0 to 0 as the complement $q$, where $q=1-p$.

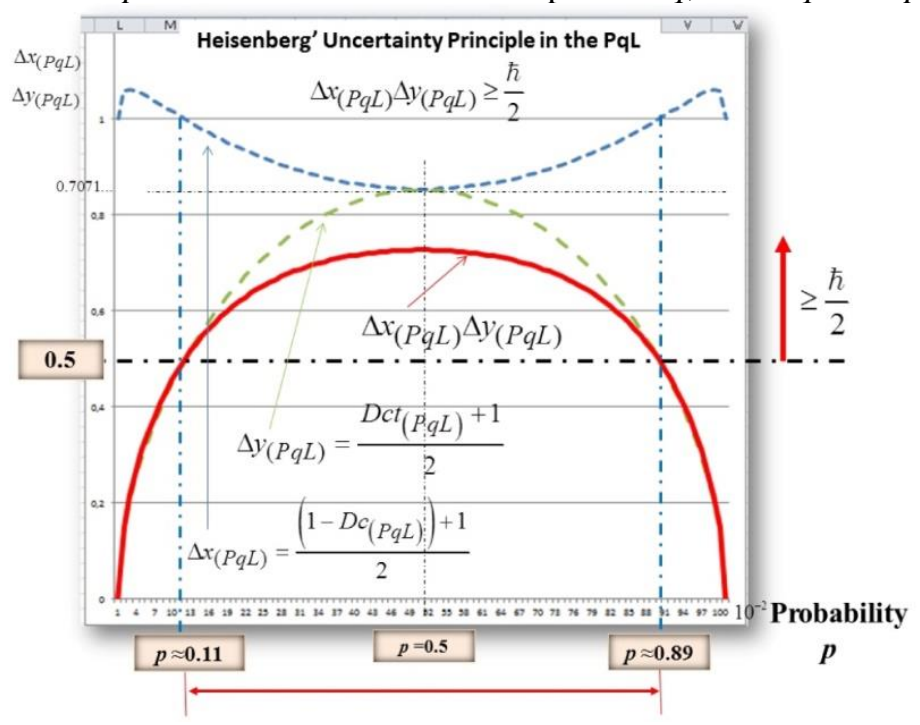

(a)

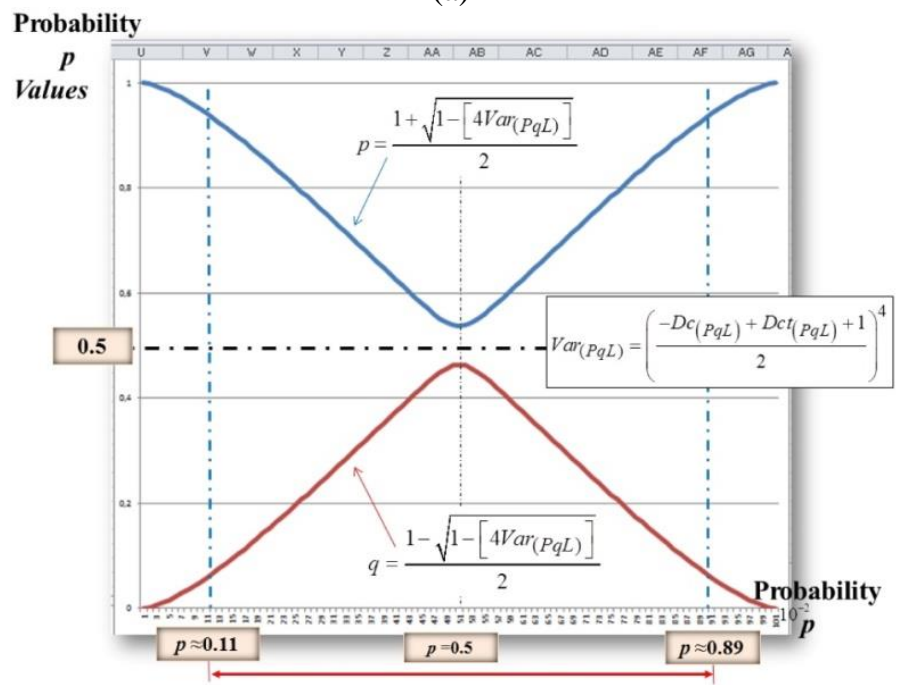

(b)

Fig. 12. Heisenberg uncertainty principle and the calculation of the probability value: (a) results of the simulation of the PqL with the Heisenberg uncertainty principle based on Eqs. (80) and (81); (b) results of the simulation based on Eqs. (84) and (85) to obtain probability values ranging from 0 to 1.0.

\subsection{Results Related to the Representation of the Division and Distance between Particles}

The divisions and distances between particles in the paraconsistent model of the atom can be represented by including factors in the probabilistic equations of the degree of certainty. Thus, a division and spacing 
between particles can be made by changing the equations of energies in Quadrant I of the interlaced PqL bilattice. These modifications in the Quadrant I will be expanded to the three other quadrants through the negation, complement, and conflation operators. In this procedure, the equations of the degree of contradiction are left unchanged, and a constant value is inserted into the equations of the degree of certainty, which we will call the distancing factor $\left(d_{f}\right)$. This causes a change of the values and locations of the probabilistic trajectories of the logical states. The new equations generated will have the same values for the degrees of evidence, which is represented by the Shannon entropy, and simulate other particles with different distances from each other, which depend on the value of $d_{f}$ inserted into the equation. The inclusion of the $d_{f}$ constant enables orbital paths or paths to be chosen in the model to simulate the bonding between atoms and also enables to carry out other studies related to sets of elementary particles.

Figure 13(a) shows the simulation of two particles in separation, with $d_{f}=1$, and Fig. 13(b) shows the simulation of two particles in separation, with $d_{f}=3$.

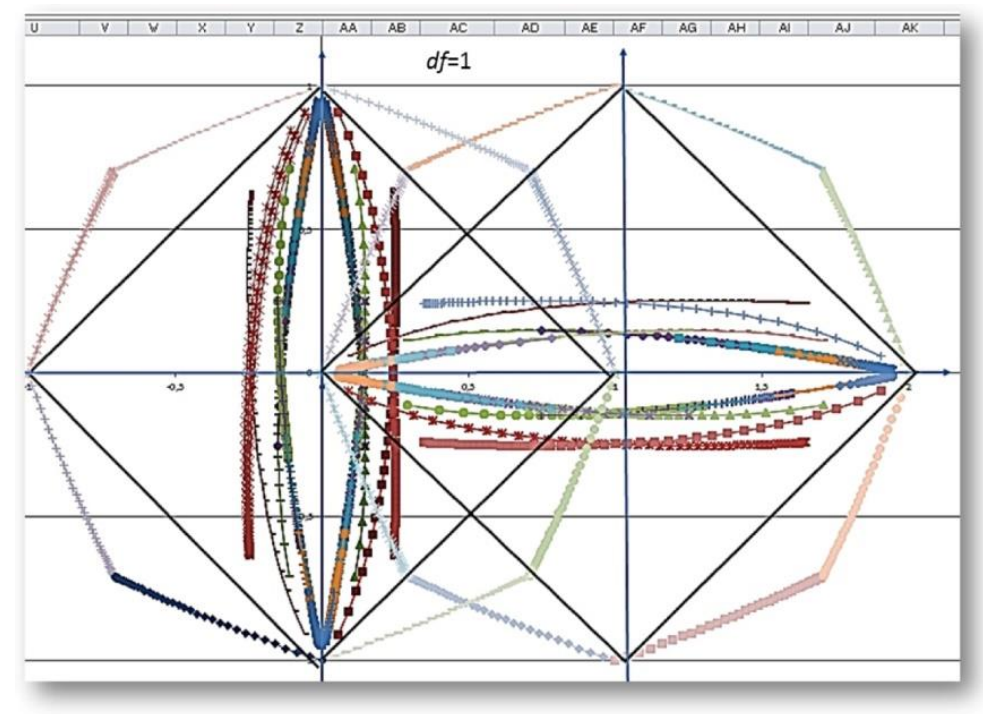

(a)

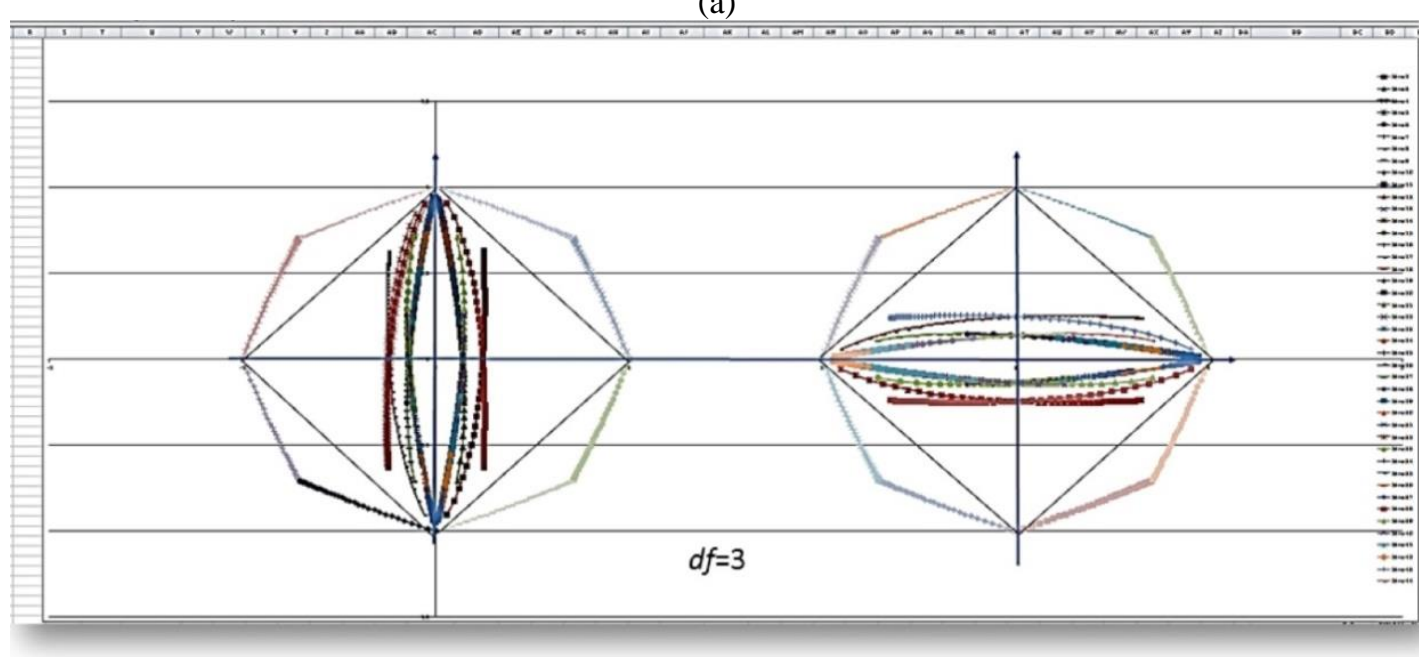

(b)

Fig. 13. Representation of the division and distance between particles: (a) simulation of two particles in separation, with $d_{f}=1$; (b) simulation of two particles in separation, with $d_{f}=3$. 


\subsection{Simulation Results with Energy Layer Values for the Hydrogen Atom}

As previously discussed, the energy values in each layer will be obtained by multiplying each value of the degree of certainty obtained in each layer of the paraconsistent model of the atom by the value of the groundstate energy of the hydrogen atom. For a better visualization of the results in the graphs, we will use in the equations the module of energy value $\left|E_{R y d}\right|=13.6 \mathrm{eV}$.

Figure 14(a) shows the simulation results for energy layers in the degenerate states of the hydrogen atom in the paraconsistent model of the atom by application of the Eqs. (34), (42), (50), (58), and (66).

\subsubsection{Adjustments in Energy Equations to Stabilize the Graphical Results in the Layers of Hydrogen Atom}

An adjustment factor $F_{a d j}$ is considered from the observation of the extreme results $\left(\geq p_{\max }\right)$ and $\left(\leq p_{\min }\right)$ obtained using Eq. (34), where the necessity of a multiplicative value to attenuate the value of the extremes by about $85 \%$ is verified. Considering that the Shannon normalization factor value is $1=1.057402554$, the $F_{a d j}$ will be the inverse of the cube of the normalization factor, such that $F_{a d j}=\frac{1}{1^{3}} ; 0.845821917$. For the energy between layers 1 and 2 , the corresponding maximum multiplicative value will be $\frac{1}{1^{3}}\left(1-\frac{1}{\sqrt{2}}\right) ; 0.247656$.

To keep in the layers the energy amplitude at constant values over the range defined by the maximum $\left(p_{\max } ; 0.89\right)$ and minimum $\left(p_{\min } ; 0.11\right)$ values of probability, we will apply the adjustment factor $F_{a d j}$ in Eqs. (34), (42), (50), (58), and (66).

From Eq. (34), the adjustment factor of $F_{a d j}=\frac{1}{1^{3}}$ is applied to the degree of certainty to determine the energy of the second layer with a constant value over the range defined by the maximum $\left(p_{(\max )}\right)$ and minimum $\left(p_{(\mathrm{min})}\right)$ values of probability. Equation (34) in this adjusted format for a hydrogen atom becomes

$$
E n_{(P q L) E 2}=\left[\frac{1}{1^{3}}\left(H_{(s) P q L}-H_{(s) P q L} \sqrt{\frac{H_{(s) P q L}}{2}}\right)\right] \times 13.6 \mathrm{eV} .
$$

From Eq. (42), the energy values of layer 3 can be computed using multiples of the adjustment factor of $\frac{1}{1^{3}}$. Equation (42) in this adjusted format for a hydrogen atom becomes

$$
E n_{(P q L) E 3}=\frac{1}{1^{5}}\left[\frac{1}{1^{6}} \sqrt{\frac{H_{(s) P q L}}{2}}-\left(\frac{1}{1^{6}} \sqrt{\frac{H_{(s) P q L}}{2}} \times \sqrt{\frac{1}{1^{6}} \sqrt{\frac{H_{(s) P q L}}{2}}}\right)\right] \times 13.6 \mathrm{eV} .
$$

From Eq. (50), the energy values of layer 4 can be computed using multiples of the adjustment factor of $\frac{1}{1^{3}}$. Equation (50) in this adjusted format for a hydrogen atom becomes

$$
E n_{(P q L) E 4}=\frac{1}{1^{16}}\left[\frac{1}{1^{6}} \sqrt{\frac{1}{1^{6}} \sqrt{\frac{H_{(s) P q L}}{2}}}-\left(\frac{1}{1^{6}} \sqrt{\frac{1}{1^{6}} \sqrt{\frac{H_{(s) P q L}}{2}}} \times \sqrt{\frac{1}{1^{6}} \sqrt{\frac{1}{1^{6}} \sqrt{\frac{H_{(s) P q L}}{2}}}}\right)\right] \times 13.6 \mathrm{eV} .
$$

From Eq. (58), the energy values of layer 5 can be computed using multiples of the adjustment factor of $\frac{1}{1^{3}}$.

Equation (58) in this adjusted format for a hydrogen atom becomes

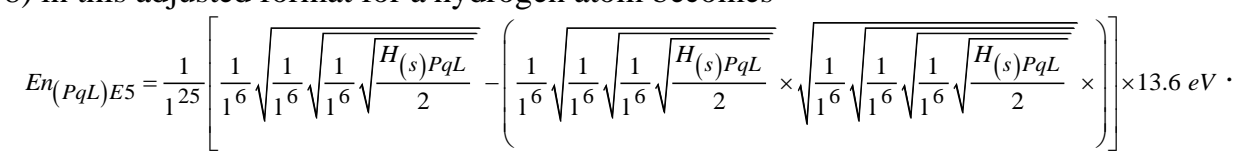

From Eq. (66), the energy values of layer 6 can be computed using multiples of the adjustment factor of $\frac{1}{1^{3}}$. 
Equation (66) in this adjusted format for a hydrogen atom becomes

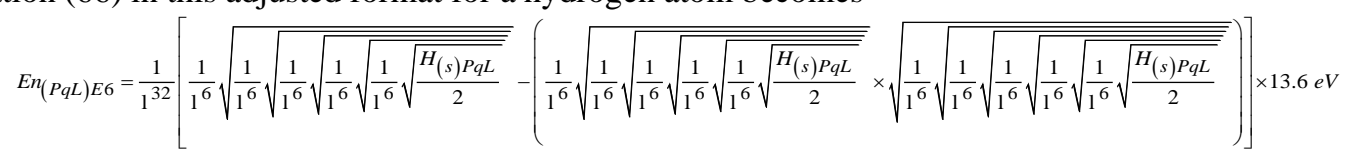

These procedures for $\mathrm{PqL}$ equations can be continued for $n$ layers of the paraconsistent model applied to the hydrogen atom.

Figure 14(b) shows the results of the paraconsistent functions of the energies obtained from the hydrogen atom using multiples of the adjustment factor of $\frac{1}{1^{3}}$.

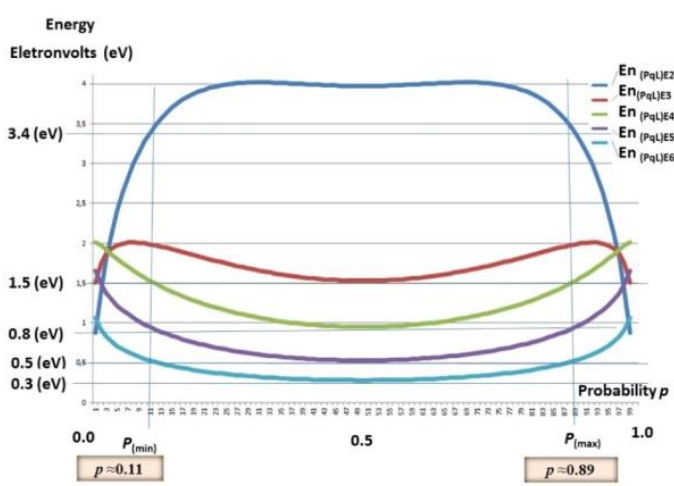

(a)

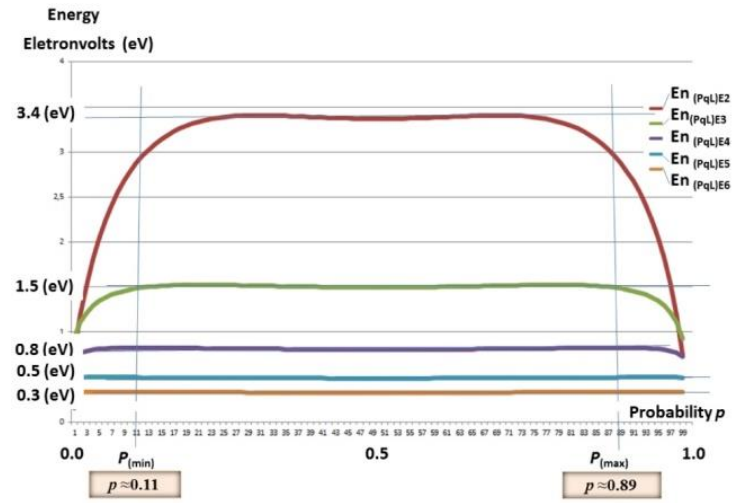

(b)

Fig. 14. Energy layer values for the hydrogen atom: (a) energy values of the layers in the paraconsistent model of the atom in the representation based on Eqs. (34), (42), (50), (58), and (66); (b) energy values of the

layers in the hydrogen atom obtained with Eqs. (86) to (90) in the application of the adjustment factor

$$
F_{\text {adj }}=\frac{1}{1^{3}} \text {. }
$$

\subsection{Results of the Paraconsistent Model of the Atom Applied to Raman Spectroscopy}

Data from Raman spectroscopy are obtained through vibrational processes of molecules involving laser application and capturing responses in the form of energy pulses. The obtained Raman information is related to the spectral lines that are provided as frequency-dominated Raman shifts or wavenumbers expressed in $\mathrm{cm}^{-1}$. In this work, we experimentally investigate how the paraconsistent model of the atom responds to variations in a particular wavelength range.

This experimental simulation involves applying the normalized values of Raman intensity in the equation of the degree of favorable evidence presented in Eq. (23). In this application, the values of probability $p$ are replaced in the Shannon entropy function by the complement of the normalized values of Raman intensity (1$I_{\text {Raman }}$ ) throughout the spectrum of the sample.

$$
\begin{aligned}
& H_{(s) P q L(k)}=-1 \pi\left[p_{n i} \log p_{n i}+\left(1-p_{n i}\right) \log \left(1-p_{n i}\right)\right] \rightarrow \\
& H_{(s) P q L(k)}=-1 \pi\left[I_{\text {Raman }(n i)} \log I_{\text {Raman }(n i)}+\left(1-I_{\text {Raman }(n i)}\right) \log \left(1-I_{\text {Raman }(n i)}\right)\right] .
\end{aligned}
$$

As $\mu_{(P q L) k}=H_{(s) P q L(k)}$, the degree of evidence, which is probabilistic, is modified and becomes the degree of evidence of Raman intensity, with spectroscopy characteristics that interfere with the energy amplitude values in the hydrogen atom layers.

Figure 15(a) shows two Raman spectroscopy signals in the 400 to $1,400 \mathrm{~cm}^{-1}$ range, which were recorded from a lubricating mineral oil sample. The first spectrum (Type 1) is related to the Raman data of normal lubricating mineral oil, and the second spectrum (Type 2) shows the Raman data of non-normal lubricating mineral oil. The Type-2 (non-normal) lubricating mineral oil had its temperature controlled and maintained at approximately $127.5^{\circ} \mathrm{C}$ for $8 \mathrm{~h}$ and cooled to room temperature to obtain the Raman sample. The two Raman data spectra were applied to the paraconsistent model of the hydrogen atom and analyzed through the representation of the energy levels of the layers presented in this work (Eqs. 86 to 90).

Figure 15(b) shows the simulation results obtained for the energy levels of the six layers in the paraconsistent hydrogen atom. In a superficial analysis, it is verified that the spectrum of the normal lubricating mineral oil (Type 1) presents variations in the layers of the atom at several wavelengths. Some parts of the spectrum of the non-normal lubricating mineral oil (Type 2) exhibit few energy variations in the layers of the atom considering the investigated spectrum range. 

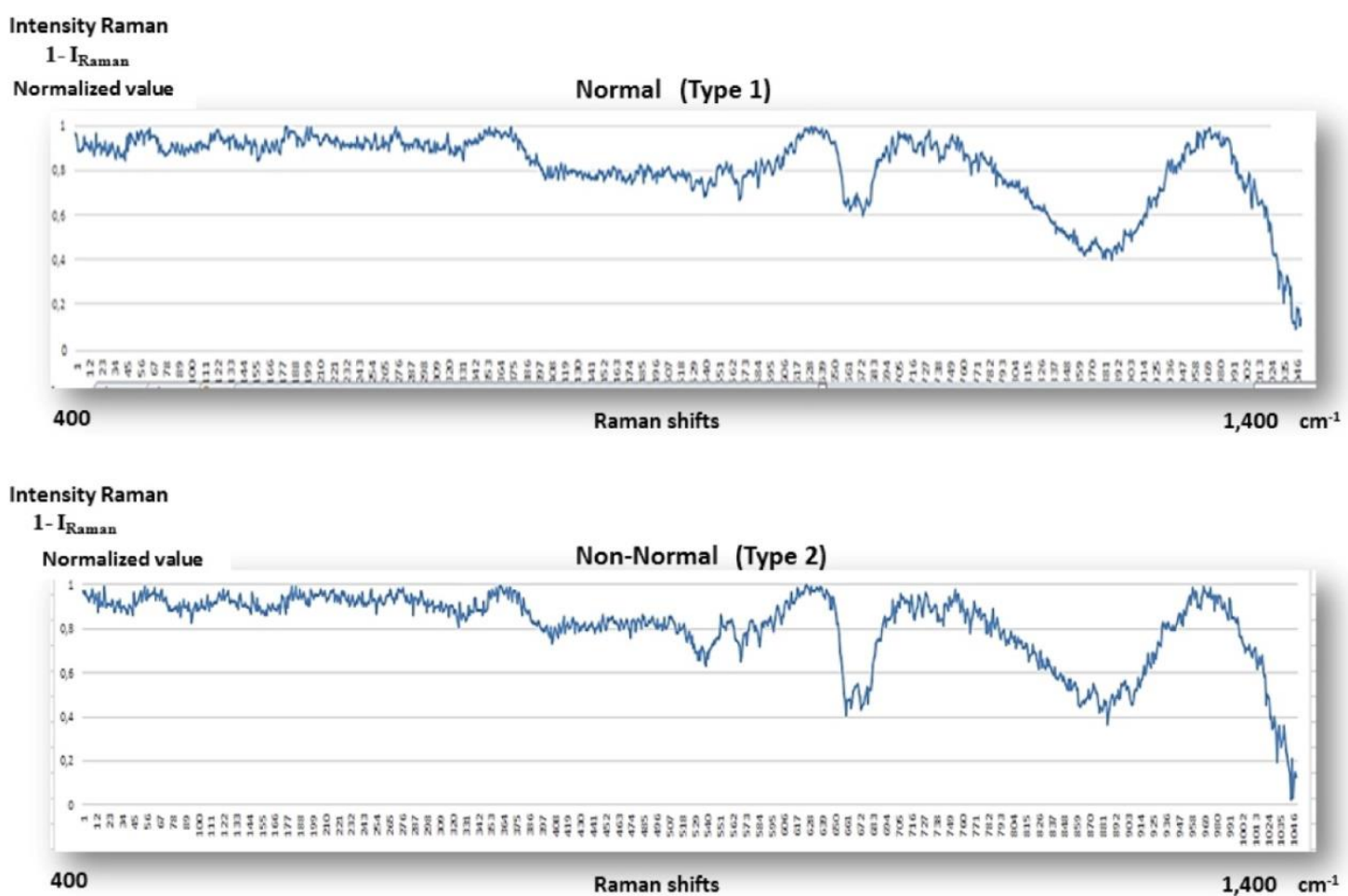

(a)

Energy levels in the layers of paraconsistent hydrogen atom

x $13.6(\mathrm{eV})$

Normal (Type 1)

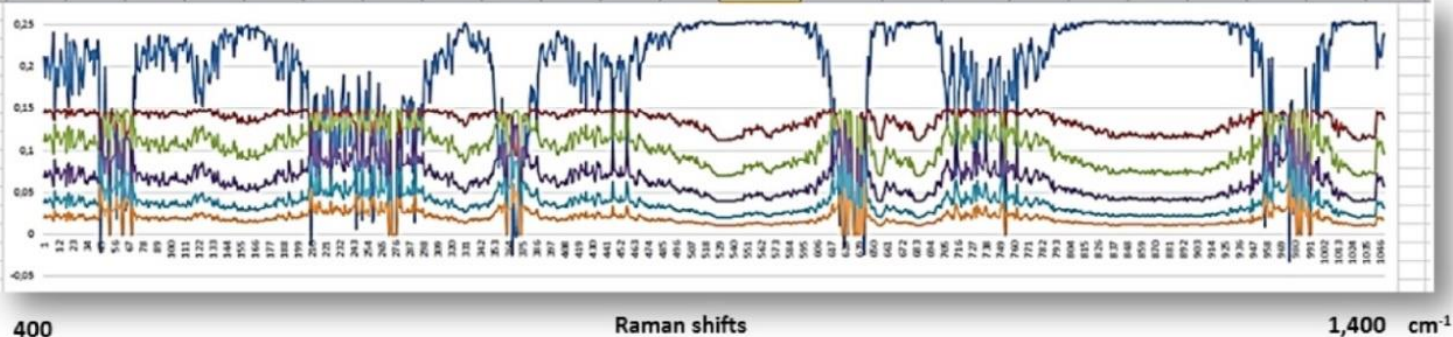

Energy levels in the layers of paraconsistent hydrogen atom

$\mathrm{x} 13.6(\mathrm{eV}) \quad$ Non-Normal (Type 2)

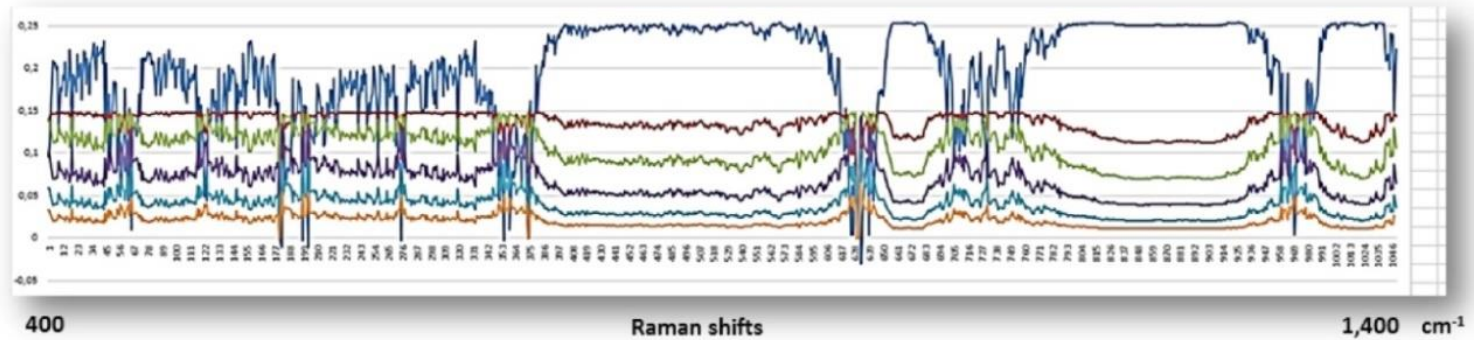

(b)

Fig. 15. Practical example results of the paraconsistent model of the atom applied using Raman spectroscopy analysis: (a) Raman spectroscopy signals from a lubricating mineral oil sample considered normal (Type 1) and non-normal (Type 2; heated for a preset time) (in 1- $\mathrm{I}_{\text {Raman }}$ normalized values); (b) simulation results obtained for the energy levels of the six layers in the paraconsistent hydrogen atom. 


\section{Discussion}

The equations presented in this work, as well as the method of obtaining them through interpretations of the interlaced PqL bilattice, follow the fundamentals of PAL2v, where the degree of favorable evidence $\mu$ must be accompanied by the degree of unfavorable evidence $\lambda$ to form the annotation. In the same manner, the degree of certainty $D c$ must be accompanied by the degree of contradiction $D c t$ to form the paraconsistent logical state.

In Fig. 6(a), the graphs of the results obtained by simulations with Shannon entropy show how the variation of probability $p$ creates the path of logical states within the interlaced PqL bilattice. Notably, the correlation value of 0.5 between the curves of the degrees of evidence is the point that defines the boundaries $p_{(\min )}=0.11$ and $\left.p_{(\max )}=0.89\right)$ between the evolution of the states in a balanced quantum system and the collapse of the wave function with the definition of a false or true final logical state.

Figure 6(b) shows the unit value of the modulus of the internal vector $\mathrm{P} \psi_{\text {int }}$ that moves to create a geometric arc. The limits of its slope are defined by the equations of the Shannon entropy, and its movement with the two external vectors created by the complementarity of values indicates the uncertainty in the movement directions of these vectors.

The paraconsistent model of the atom presents the uncertainties that lead to incompleteness in the measurements, which is expected of a quantum system. This was demonstrated in the results of the simulations shown in Figs. 7(a) and 7(b), where the movements of the external vectors are antagonistic.

In Figs. 8 and $\mathbf{9}$, the results show the models separated by the reference of two observers.

Figure 10 shows the simulation results for the complete paraconsistent model of the atom, as well as its projection of the state vectors in the imaginary $y$-axis of the contradiction and in the real $x$-axis in the case of an effected measurement. It was demonstrated how the actions of the negation, complementation, and conflation operators applied to the logical states enabled the expansion of the probability values. From the analysis, the results obtained by the application of the operators to the logical states of Quadrant I show that the probabilistic trajectories of the particles in the layers appear simultaneously in two directions, that is, clockwise and counterclockwise.

For a practical interpretation, the external and internal probabilistic trajectories of the paraconsistent model of the atom mean that the $\mathrm{PqL}$ operations (negation, complementation, and conflation) occur simultaneously, acting in four quadrants of the interlaced PqL bilattice. For an observer in $\mathrm{X}$, in the first quadrant, each paraquantum logical state located at the end of the external state vector ( $\left.\mathrm{P} \psi_{\text {ext }}\right)$ simultaneously generates a paraquantum logical state located at the end of the complementary external state vector ( $\left.\mathrm{P} \psi_{\text {Cext }}\right)$.

This is also the same for a $Y$ observer; thus, four paraquantum logical states are generated in Quadrant I. Therefore, each single logical state of the paraconsistent model of the atom is, in the reality, composed of eight paraquantum logical states. As each paraquantum logical state has one equation for the degree of certainty and one equation for the complement of the degree of contradiction, then 16 equations are used for this representation.

In the construction of the model, each of these PqL equations receives the probabilistic value $p$ and its complement $(1-p)$ so that through the Shannon entropy functions they can present the paraconsistent results. The variations of the probabilistic values, and the frequency in which they are applied in the PqL equations result in the trajectories presented in the paraconsistent model of the atom. The countless PqL-equations of 
the paraquantum logical states obtained in the atom layers simultaneously generate all the internal trajectories that are presented in the paraconsistent model. In the paraconsistent model of the atom, the internal state

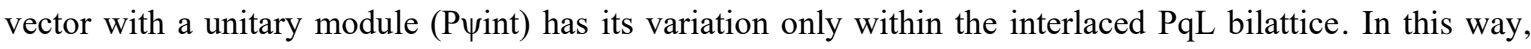
this internal vector, Pyint, supports all formalization of PqL through the paraquantum logical states located at its end.

The external state vector (P $\psi$ ext), in turn, exceeds the limits of the interlaced bilattice and represents energy values through its paraquantum logical states located at its end. In this way, the actions of these two vectors show through the probabilistic trajectories of the particles in the paraconsistent model of the atom a clear interface between the logical universe and the real/quantum world.

Figures 11(a) and 11(b) show the simulation results of the wave functions for the ground-state energy layer and the wave functions for the other internal energy layers. These results verify the limits that define the evolution of the states.

Figure 12(a) illustrates the validation of the results of the simulation with the Heisenberg uncertainty principle in the PqL, and Fig. 12(b) shows that is possible to obtain the value of the probability $p$ considering only the values of the degrees of certainty and contradiction. With these simulations, quantum concepts, such as probability density and wave function, are well established by the equations and probabilistic functions in the PqL. It is highlighted here that in the simulations performed to obtain the wave functions, the Heisenberg uncertainty principle equations, the probability values, and the values of the degrees of certainty and contradiction that make up the paraquantum logical states were used as input. The results demonstrate the application of the reversibility characteristic in the paraconsistent model of the atom.

The results presented in Fig. 13 show the possibility of analyzing the paraconsistent model of the atom and other concepts of quantum mechanics. The separation imposed by a distance factor, which is applied in the equations, allows simulating effects in which a separate particle remains in the perception of a $Y$ observer while the other is in the perception of an $\mathrm{X}$ observer. This condition is important as it enables the paraconsistent model of the atom capable of considered the quantum entanglement representations.

In Fig. 14, the energies of the orbital layers represented by the pairs of values are well delineated in the representation of the hydrogen atom, with values close to those obtained by the Bohr model.

On the basis of the results presented in Fig. 14, the experiments with Raman spectroscopy have been elaborated, the results of which are presented in Fig. 15. A visual analysis of the results shown in Fig. 15(b) indicates that with $\mathrm{PqL}$ equations, the differences in energy variations between a normal lubricating mineral oil (Type 1) and a non-normal lubricating mineral oil (heated at $127.5{ }^{\circ} \mathrm{C}$ for $8 \mathrm{~h}$; Type 2) can be verified. Therefore, this PqL-based technique can be useful for spectroscopic signal analysis and the verification of material properties at atomic levels.

In general, the simulation results define the probabilistic characteristics of the particle, whereby in quantum mechanics, before the measurement, any of the physical properties are always indefinite. In this manner, the configuration model exhibits the geometry of a sphere and can be described using equations that consider angular variables.

A representation of the two planes can be made according to Fig. 12, where the orthogonality of the two planes forms an octahedron in which an analysis of the external and internal variables in the equations validates the variation of probability. With the probability representation obtained through the PqL equations 
in which the Shannon entropy is introduced, we can derive the equations of the degrees of certainty and contradiction by applying the conditions used for a paraconsistent Bloch sphere as an example.

\section{Conclusions}

The paraconsistent model of the atom proposed in this work is based on the foundations of the PAL and combines the concepts of the entropy of information theory with quantum mechanics. The proposed paraconsistent model of the atom, which applies the interlaced PqL bilattice, shows a convincing geometric aspect for the atomic particle. Thus, well-adjusted fundamentals for the phenomena of quantum physics could be demonstrated. Despite the impossibility of covering all quantum phenomena in this work, the equations obtained by the analyses provide the characteristics of symmetry, recurrence, and superposition of states. The entanglement concept can be expressed in the form of the division obtained through the distance factor $d_{F}$ used in the equations of the degrees of certainty. The quantum phenomenon of the superposition of states is explicitly expressed through the equations and the representation in which two observers are active. This procedure, which was conducted through the simultaneous use of equations and the analysis of an observer in the vector of the base $X$ and an observer in the vector of the base $Y$, is similar to the quantum theory. The model presented in this work is innovative and opens a field of in-depth investigations of different conditions and the effects originating from interpretations of the model under diverse conditions and dimensions. All the equations feature good computability and ensure that all the procedures can be presented in matrix and algorithmic forms. Notably, in the presented paraconsistent model of the atom, no equation results in a defined value. In general, all the equations used in the simulations are probabilistic functions that result in indefinite trajectories of paraquantum logical states. Even the direction and orientation of the orbital trajectories shown in the result graphics are indefinite because they are always opposite to one another. In the simulations, the paraconsistent model of the atom was represented by algorithms I and II that allowed showing the probabilistic trajectories of the paraquantum logical states that can be related to the probable orbits of the elementary particles. With the simulation applying the PqL equations, the energy functions, represented by the degrees of certainty and contradiction, therefore values generated in the quantum universe - Interlaced PqL Bilattice -, can to express the probabilities to be measured in the real world. These algorithms and equations have high significance for the beginning of new studies in computational applications of PqL. In its application to Raman spectroscopy data, the paraconsistent model showed versatility and a good representation of the energy variations at the atomic level of hydrogen. These results enable us to further explore the use of the proposed model with the presented concepts, such as the adjustment of the energy values in the lower layers of the hydrogen atom and for other atomic models. This work demonstrates that the use of the Shannon entropy in a paraconsistent model is an excellent method for modeling quantum systems. In the future, new simulation procedures will expand the application possibilities of the paraconsistent model of the atom in other areas, including computation, quantum logic gates, quantum systems for signal recognition, and quantum cryptography.

Supplementary Materials: The data that support the plots within this paper and other findings of this study are available in additional material and from the corresponding author.

Author Contributions: J.I. carried out the experiments, did the analysis of the results, wrote the paper and prepared the illustrations. 
Acknowledgments:

The author acknowledges ISESC - Instituto Superior de Educação Santa Cecilia for the financial support for this research.

The author would like to acknowledge L. Silveira Jr. for Raman spectroscopy data obtained by FAPESP (São Paulo Research Foundation) process no. 2009/01788-5.

Conflicts of Interest: The author declares no competing interests.

\section{References}

[1] Kragh, H.; Rigden, J. S. Niels Bohr and the Quantum Atom: The Bohr Model of Atomic Structure 19131925. American Journal of Physics 81(3):237-238, 2013. DOI: $10.1119 / 1.4771884$

[2] Heilbron, J. L.; Kuhn, T. S. The genesis of the Bohr atom. Historical Studies in the Physical Sciences 1, 211-290, 1969. DOI: $10.2307 / 27757291$

[3] Auletta, G. Foundations and Interpretation of Quantum Mechanics, World Scientific, 2001.

[4] Gribbin, J. Erwin Schrodinger and the Quantum Revolution. Ed. Random House ISBN1446465713, 9781446465714, 2012.

[5] Bohm, D. A. Suggested Interpretation of the Quantum Theory in Terms of "Hidden" Variables, Phys. Rev. 85, 166-193, 1952.

[6] Feynmann, R. P. The Feynman Lectures on Physics, Volume 3, Addison Wesley, 1963.

[7] Feynmann, R. P. Simulation physics with Computers, Journ. Th. Phys. 21, 467, 1982.

[8] Dirac, P. A. M. The Principles of Quantum Mechanics, 16 Oxford University Press, 1958.

[9] Born, M. The statistical interpretation of quantum mechanics - Nobel Lecture, December 11, 1954.

[10] Nielsen, M. A.; Chuang, I. L. Quantum Computation and Quantum Information. Cambridge University Press, 2000.

[11] von Neumann, J. Applications of the ergodic hypothesis. Proceedings of the National Academy of Sciences 18, 263-266. Reprinted in (Taub 1961a) 13. 1932.

[12] Engesser, K.; Gabbay, D. M.; Lehmann, D. A. New Approach to Quantum Logic (Studies in Logic book 8). 200p. ISBN-13: 978-1904987536. College Publications, 2007.

[13] Aspray, W. The mathematical reception of the modern computer: John von Neumann and the institute for advanced study computer. In Studies in the History of Mathematics, E. R. Phillips, Ed., v. 26. MAA, Washington DC, pp. 166-194, 1987.

[14] Bruckmann, G.; Weber, W. Eds. Contributions to the von Neumann Growth Model (New York), Springer Verlag, 1971.

[15] Da Costa, N. C.; de Ronde, A. C. Found Phys 43: 845, 2013, DOI: 10.1007/s10701-013-9721-9.

[16] Bueno-Soler, J.; Carnielli W. Paraconsistent Probabilities: Consistency, Contradictions and Bayes' Theorem. Entropy 18(9), 325, 2016 ; https://doi.org/10.3390/e18090325

[17] Da Costa, N. C. A. On the theory of inconsistent formal systems. NotreDame Journal Form Log, 15(4), 497-510. 1974. DOI: http://dx.doi.org/10.1305/sdjfl/1093891487

[18] Abe, J. M.; Nakamatsu, K.; Akama, S.; Da Silva Filho, J. I. The Importance of Paraconsistency and Paracompleteness in Intelligent Systems. In: Czarnowski I., Howlett R., Jain L. (eds) Intelligent Decision Technologies 2017. IDT 2017. Smart Innovation, Systems and Technologies, 73. Springer, Cham, (2018).

[19] Da Costa, N. C. A.; Marconi, D. An overview of Paraconsistent logic in the 80's., The Journal of NonClassical Logic, 6, 5-31, 1989.

[20] Abe, J. M.; Akama, S.; Nakamatsu, K. Introduction to Annotated Logics. Springer, Heidelberg, 2016

[21] Blair, H. A.; Subrahmanian, V. S. Paraconsistent Logic Programming, Theoretical Computer Science 68, 135-154, 1989.

[22] Belnap, N. A. Useful four-valued logic. In J. M. Dunn \& G. Epstein (orgs.), Modern uses of multiplevalued logic (p. 8-37). Dordrecht: Reidel. 1977b.

[23] Belnap, N. How a computer should think. In G. Ryle (ed.), Contemporary Aspects of Philosophy (pp. 30-55). Stocks_eld: Oriel Press. 1977a.

[24] Subrahmanian, V. S. On the semantics of quantitative logic programs. Proc. 4th. IEEE Symposium on Logic Programming, Computer Society Press, Washington D.C, 1987.

[25] Da Silva Filho, J. I. Interpretation methods of Paraconsistent Annotated logic with annotation of two values - LPA2v with construction of algorithm and implementation of Electronic Circuits. Doctoral. Thesis (in portuguese), Polytechnic School of the University of São Paulo, POLI / USP São Paulo, Brazil, p. 115, 1999. 
[26] Da Silva Filho J. I.; Lambert-Torres, G.; Abe J. M. Uncertainty Treatment Using Paraconsistent Logic Introducing Paraconsistent Artificial Neural Networks. IOS Press, 328 Volume 211- Frontiers in Artificial Intelligence and Applications, Amsterdam, Netherlands, 2010.

[27] Garcia, D. V.; Da Silva Filho, J. I.; Silveira Jr, L. et al., Analysis of Raman spectroscopy data with algorithms based on paraconsistent logic for characterization of skin cancer lesions. Vibrational Spectroscopy, 103, 2019. DOI: 10.1016/j.vibspec.2019.102929

[28] Da Silva Filho, J. I.; Nunes, V. C.; Garcia, D. V.; Mario, M.C.; Giordano, F.; Abe, J. M.; Pacheco, M. T. T.; Silveira Jr., L. Paraconsistent analysis network applied in the treatment of Raman spectroscopy data to support medical diagnosis of skin cancer. Med. Biol. Eng. Comput. 54, 1-15, 2016). DOI: $10.1007 / \mathrm{s} 11517-016-1471-3$

[29] Coelho, M. S.; Da Silva Filho, J. I.; Côrtes, H. M. et al. Hybrid PI controller constructed with paraconsistent annotated logic. Control Engineering Practice, 84, 112-124, 2019. DOI: 10.1016/j.conengprac.2018.11.007

[30] Misseno Da Cruz, C.; Rocco, A.; Mario, M.C.; Garcia, D.V.; Lambert-Torres, G.; Abe, J.M.; Torres, C.R.; Da Silva Filho, J.I. Application of Paraconsistent Artificial Neural Network in Statistical Process Control acting on voltage level monitoring in Electrical Power Systems. 18th International Conference on Intelligent System Application to Power Systems, ISAP 201510 November 2015, Porto; Portugal; 11 September 2015 through 17 September 2015; Category number CFP15755-USB; Code 118601, 2015.

[31] Da Silva Filho, J. I. A Probabilistic Paraconsistent Logical Model for Non-Relativistic Quantum Mechanics Using Interlaced Bilattices with Conflation and Bernoulli Distribution. Journal of Quantum Information Science, 7, 89-124. 2017. DOI: 10.4236/jqis.2017.73009

[32] Da Silva Filho, J. I. Undulatory Theory with Paraconsistent Logic (Part I): Quantum Logical Model with Two Wave Functions. Journal of Quantum Information Science, 6, 143-180, 2016. DOI: 10.4236/jqis.2016.63012

[33] Da Silva Filho, J. I. Undulatory Theory with Paraconsistent Logic (Part II): Schrödinger Equation and Probability Representation. Journal of Quantum Information Science, 6, 181-213, 2016. DOI: 10.4236/jqis.2016.63013

[34] Ginsberg, M. L. Multivalued logics: A uniform approach to inference in artificial intelligence, Computational Intelligence 4, 265-316, 1988.

[35] Shannon, C. E. Bell Syst. Tech. J. $271948379\{423$ and 623\{656, DOI:10.1002/j.15387305.1948.tb01338.x

[36] Shannon, C. E.; Weaver W. The Mathematical Theory of Communication, Illini Books, Illinois, 1949.

[37] Tempesta, P. Beyond the Shannon-Khinchin formulation: The composability axiom and the universalgroup entropy. Annals of Physics, 365, 180-197, 2016. DOI: 10.1016/j.aop.2015.08.013

[38] Vedral, V. The role of relative entropy in quantum information theory. Rev. Mod. Phys. 74, $197,2002$.

[39] Nielsen, M. A., Chuang, I. L. Quantum Computation and Quantum Information. Massachusetts Institute of Technology, 702 pages - 2010, ISBN: 9781107002173

[40] Rydberg, J.R. Den Kungliga Svenska Vetenskapsakadem-iens Handlingar 23 (11), 1889.

[41] Nedelman, J. and Wallenius, T. Bernoulli Trials, Poison Trials, Surprising Variances, and Jensens's Inequality. The American Statistician, 40, 286-289. 1986. 